\title{
RIBBON TORUS KNOTS PRESENTED BY VIRTUAL KNOTS WITH UP TO 4 CROSSINGS
}

\section{ATSUSHI ICHIMORI AND TAIZO KANENOBU}

\begin{tabular}{|c|l|}
\hline Citation & OCAMI Preprint Series \\
\hline Issue Date & 2010 \\
\hline Type & Preprint \\
\hline Textversion & Author \\
\hline Rights & $\begin{array}{l}\text { C } 2012 \text { World Scientific Publishing Company. For personal use only. No other } \\
\text { uses without permission. }\end{array}$ \\
\hline Relation & $\begin{array}{l}\text { Preprint of an article submitted for consideration in Journal of Knot Theory and } \\
\text { Its Ramifications } \mathbb{C} 2012 \text { World Scientific Publishing Company. } \\
\text { https://www.worldscientific.com/worldscinet/jktr . }\end{array}$ \\
\hline Is version of & $\underline{\text { https://doi.org/10.1142/S0218216512400056 }}$ \\
\hline
\end{tabular}

From: Osaka City University Advanced Mathematical Institute http://www.sci.osaka-cu.ac.jp/OCAMI/publication/preprint/preprint.html 


\title{
RIBBON TORUS KNOTS PRESENTED BY VIRTUAL KNOTS WITH UP TO 4 CROSSINGS
}

\author{
ATSUSHI ICHIMORI AND TAIZO KANENOBU
}

\begin{abstract}
A ribbon torus knot embedded in the 4-space is presented by a welded virtual knot through the Tube operation due to Shin Satoh. We make an attempt of classification of ribbon torus knots presented by virtual knots with up to 4 crossings, where we use the list of virtual knots enumerated by Jeremy Green. We mainly investigate the groups of virtual knots.
\end{abstract}

\section{INTRODUCTION}

A ribbon torus knot is an embedded torus in the 4 -space $\mathbf{R}^{4}$ that is the result of piping together standardly embedded, separated 2-spheres in $\mathbf{R}^{4}$. Shin Satoh [26] has defined a Tube operation, which gives a map from a welded virtual knot $K$ to a ribbon torus knot Tube $(K)$; a welded virtual knot is an equivalence class of virtual knot allowing one of the forbidden moves $\Phi_{t}$ (Fig. 6). Satoh has also shown that this map is surjective. For a virtual knot $K$ the group $G(K)$ (see Subsec. 2.4) is an invariant for a welded virtual knot, which is isomorphic to the group of Tube $(K) ; G(K) \cong \pi_{1}\left(\mathbf{R}^{4}-\right.$ Tube $\left.(K)\right)$. In particular, if $K$ is a classical knot, $G(K)$ is the fundamental group of the complement of $K$, and so $G(K) \cong G\left(K^{*}\right)$, where $K^{*}$ is the mirror image of $K$, that is, a diagram of $K^{*}$ is obtained from that of $K$ by switching the positive to negative crossings, and vice versa. However, if $K$ is not a classical knot, then $G(K) \cong G\left(K^{*}\right)$ does not always hold. This means that $\operatorname{Tube}(K)$ and $\operatorname{Tube}\left(K^{*}\right)$ generally represent different ribbon torus knots.

On the other hand, Jeremy Green [5] has enumerated virtual knots with up to 6 real crossings. The first author [9] has calculated the groups of virtual knots with up to 4 crossings in Green's table, and has shown that each of them is isomorphic to either the infinite cyclic group or 9 groups $G_{i}, i=1, \ldots, 9$ given in Sec. 4 . In Green's table there are 9 knots with up to 3 real crossings and 108 knots with 4 real crossings, where we treat the mirror images and reversions as the same knot. However, in order to examine the group of a virtual knot we should calculate the groups of both the original knot and

Date: January 28, 2011.

Key words and phrases. Ribbon torus knot; virtual knot; group of a virtual knot; welded virtual knot.

2010 Mathematics Subject Classification. Primary 57M25.

The second author was partially supported by Grant-in-Aid for Scientific Research (C) (No. 21540092), Japan Society for the Promotion of Science. 
its mirror image. In this paper, we only investigate the ribbon torus knots represented by virtual knots with up to 4 crossings whose group are not infinite cyclic. Notice that it is conjectured that a knotted torus in the 4-space whose group is infinite cyclic is unknotted. The result is summarized in Table 1; at most nontrivial 13 ribbon torus knots arise from virtual knots with up to 4 crossings. For each group $G_{i}$, we examine all the ribbon torus knots $\operatorname{Tube}(K)$, where $K$ is a virtual knot with up to 4 crossings with group $G_{i}$. In many cases, the Gauss diagrams are useful for proving the equivalence as oriented welded virtual knots (Example 2.4, Proposition 3.4). Eventually, we see there are two pairs of ribbon torus knots with the same group $\left\{\mathrm{T} 3_{1}, \mathrm{~T} 4_{2}\right\},\left\{\mathrm{T}_{3}, \mathrm{~T} 4_{4}\right\}$, which are distinguished by the peripheral structure, and also there are two pairs we cannot decide whether they are equivalent or not; $\left\{\mathrm{T} 4_{8}, \mathrm{~T} 4_{9}\right\},\left\{\mathrm{T} 4_{10}, \mathrm{~T} 4_{11}\right\}$.

It is known that a ribbon torus knot is (-)amphicheiral, that is, the mirror image of a ribbon torus knot is equivalent to its reversion (Proposition 3.2), and so we call a ribbon torus knot symmetric if it is reversible or (+)amphicheiral (Definition 1). For each ribbon torus knot in Table 1 we examine if it is symmetric or not.

This paper is organized as follows: In Sec. 2, we give definitions of a virtual knot, a Gauss diagram, a welded virtual knot, and the group of a virtual knot. In Sec. 3, we review Satoh's Tube operation. In Sec. 4, we give our main result (Theorem 4.1), the classification of ribbon torus knots arising from virtual knots with up to 4 crossings, which is summarized in Table 1. In Secs. 5-12, we examine each ribbon torus knot given in Table 1. Sec. 13 contains some remarks and questions.

\section{VirTual KnOt and Gauss diagram}

2.1. Virtual knot. A virtual knot diagram is a knot diagram which may have virtual crossings as well as classical crossings. A virtual knot is an equivalence class of virtual knot diagrams under the classical Reidemeister moves $\Omega_{1}, \Omega_{2}, \Omega_{3}$ (Fig. 1) together with virtual Reidemeister moves $\Omega_{1}^{\prime}, \Omega_{2}^{\prime}, \Omega_{3}^{\prime}, \Omega_{4}^{\prime}$ (Fig. 2). By a generalized Reidemeister move we mean either a classical or virtual Reidemeister move. Two oriented virtual knot diagrams $D$ and $D^{\prime}$ are oriented equivalent, denoted by $D \sim D^{\prime}$, if they are related by a finite sequence of generalized Reidemeister moves.
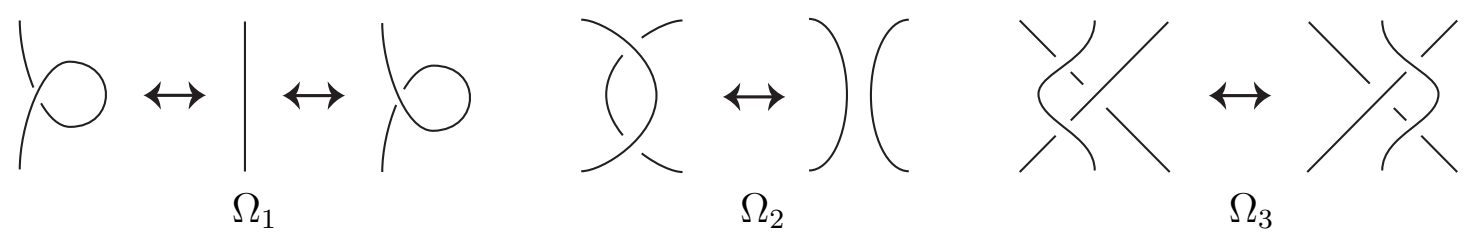

Figure 1. Classical Reidemeister moves.

Let $D$ be a diagram of an oriented virtual knot $K$. There are two mirror images: the vertical mirror image $D^{*}$ and horizontal mirror image $D^{\dagger}$; the former is obtained from $D$ by switching positive to negative crossings, and vice versa, and leaving virtual crossings unchanged, and the latter by reflecting the diagram across a vertical plane. 

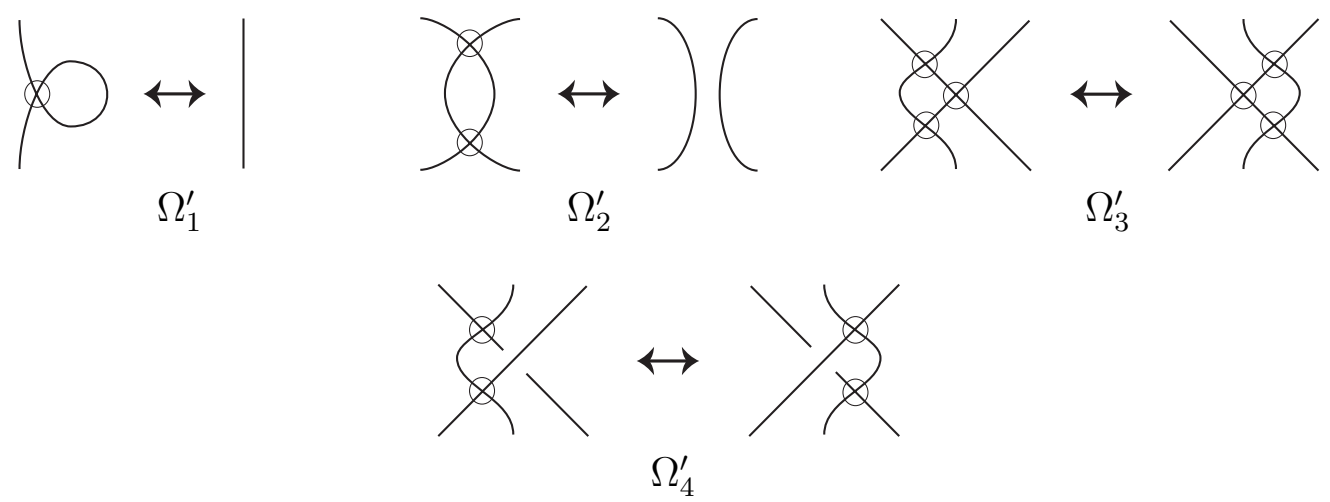

FiguRE 2. Virtual Reidemeister moves.

We denote the orientation-reversion of $D$ by $-D$. We also denote by $K^{*}, K^{\dagger}$, and $-K$ the virtual knots presented by the diagrams $D^{*}, D^{\dagger}$, and $-D$, respectively. We say a virtual knot $K$ is reversible if $K=-K$. If $K$ is a classical knot, then $K^{*}=K^{\dagger}$. However, for a virtual knot it is not always the case:

Example 2.1. In Fig. 3 we give eight oriented virtual knot diagrams, where $D$ is a diagram of the virtual knot 3.1 in Green's notation [5]. Kishino and Satoh [20, Sec. 5] have shown that these eight virtual knot diagrams are mutually inequivalent.

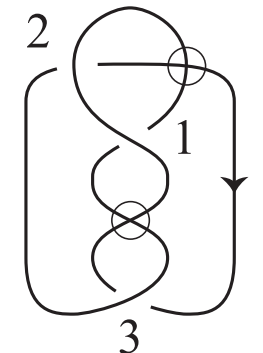

$D$

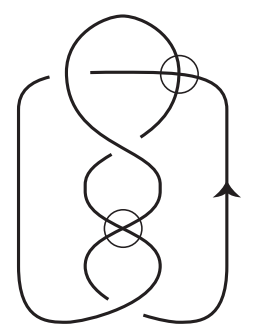

$-D$

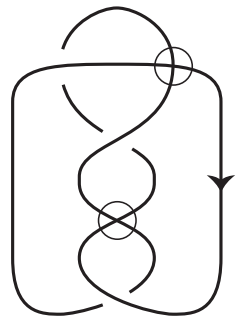

$D^{*}$

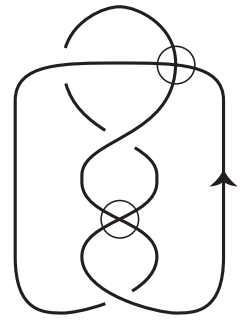

$-D^{*}$

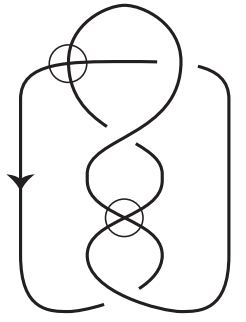

$D^{\dagger}$

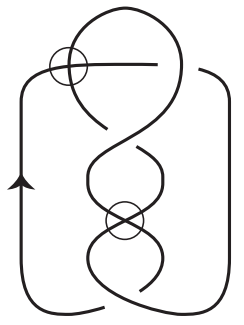

$-D^{\dagger}$

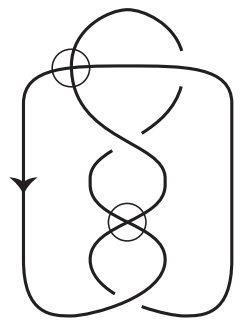

$D^{* \dagger}$

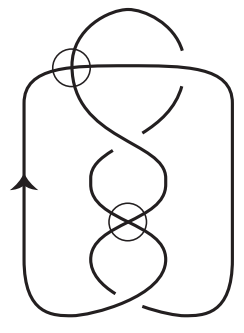

$-D^{* \dagger}$

FiguRE 3. Diagrams of the virtual knot 3.1 and its relatives. 
Remark 2.2. The terminology of vertical and horizontal mirror images follows Green [5]. The notations $D^{*}$ and $D^{\dagger}$ are different from those used in $[8,20,27,30]$.

2.2. Gauss diagram. A Gauss diagram for an oriented virtual diagram $D$ consists of the oriented circle together with chords, each of which connecting the preimages of each classical crossing point; each chord is oriented from the preimage of the overcrossing to that of the undercrossing, and further equipped with the sign of the corresponding crossing point. We assume that the circle of a Gauss diagram is oriented anticlockwise. It is known [4, Theorem 1.A] that a virtual knot is uniquely defined by the Gauss diagram. A Gauss diagram is considered up to orientation preserving homeomorphism of the underlying circle. This means that a virtual knot is equivalent to the corresponding Gauss diagram considered up to moves which are the counter-parts of Reidemeister moves for Gauss diagrams [4, Fig. 6]; we denote the corresponding moves of the Gauss diagrams by the same symbols $\Omega_{1}, \Omega_{2}, \Omega_{3}$. In particular, we will use the move corresponding to the Reidemeister move $\Omega_{1}$, see Fig. 4 .

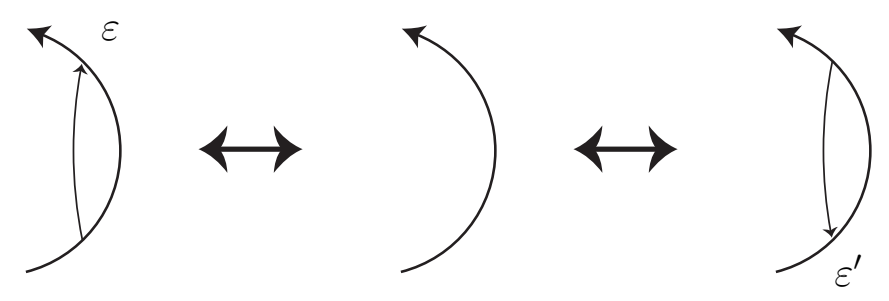

Figure 4. Moves of Gauss diagrams corresponding to Reidemeister move $\Omega_{1}$, where $\varepsilon, \varepsilon^{\prime}=+$ or - .

For an oriented virtual knot diagram $D$, we denote its Gauss diagram by $\operatorname{Gau}(D)$. Then the Gauss diagrams of $D^{*}, D^{\dagger},-D$ are obtained from that of $D$ in the following manner: $\operatorname{Gau} \beta\left(D^{*}\right)$ is obtained by changing both the orientation and the sign of each chord; Gauß $\left(D^{\dagger}\right)$ is obtained by changing the sign of each chord; Gauß $(-D)$ is obtained by turning over the whole Gauss diagram, respectively. Similarly, for a Gauss diagram $\Gamma$ we denote by $\Gamma^{*}, \Gamma^{\dagger},-\Gamma$ the Gauss diagrams obtained from $\Gamma$ by transforming in the same way as above: $\Gamma^{*}$ is obtained by changing both the orientation and the sign of each chord; $\Gamma^{\dagger}$ is obtained by changing the sign of each chord; $-\Gamma$ is obtained by turning over the whole Gauss diagram, respectively. Thus, if $\Gamma=\operatorname{Gau} \beta(D)$, then $\Gamma^{*}=\operatorname{Gauß}\left(D^{*}\right), \Gamma^{\dagger}=\operatorname{Gauß}\left(D^{\dagger}\right),-\Gamma=\operatorname{Gauß}(-D)$.

Example 2.3. In Fig. 5 we give Gauss diagrams of the eight knot diagrams given in Example 2.1, where $\operatorname{Gau}(D)$ is a Gauss diagram for the knot diagram $D$ of the knot 3.1; the Gauss code of this knot is $O 1-O 2-U 1-O 3+U 2-U 3+$; see [5].

Green [5] has enumerated virtual knots with up to 6 classical crossings by using a computer under the supervision of Dror Bar-Natan; the program generates a list of Gauss codes, and then determined which are equivalent. For each oriented virtual knot 


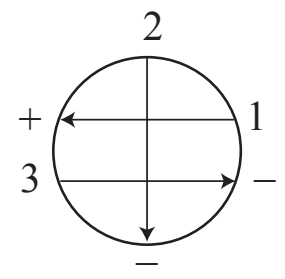

$\operatorname{Gau}(D)$

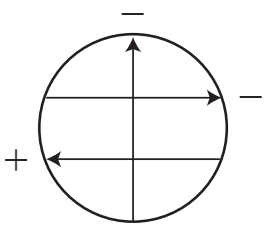

$\operatorname{Gauß}(-D)$

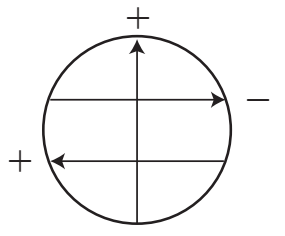

$\operatorname{Gau} \beta\left(D^{*}\right)$

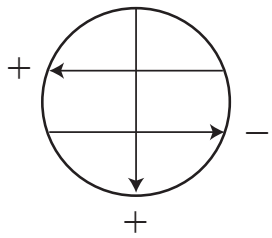

$\operatorname{Gauß}\left(-D^{*}\right)$

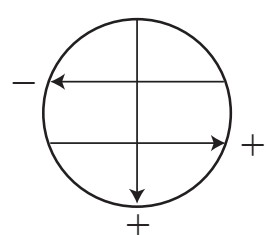

$\operatorname{Gauß}\left(D^{\dagger}\right)$

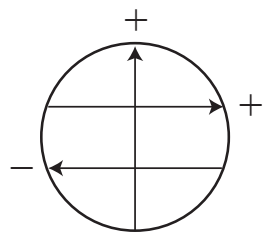

$\operatorname{Gau}\left(-D^{\dagger}\right)$

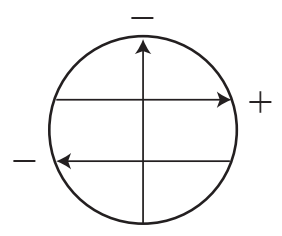

$\operatorname{Gauß}\left(D^{* \dagger}\right)$

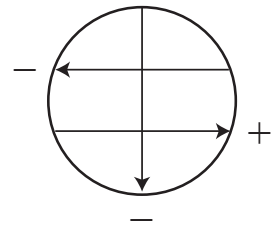

$\operatorname{Gauß}\left(-D^{* \dagger}\right)$

Figure 5. Gauss diagrams of the virtual knot diagrams given in Fig. 3.

$K$, it is also indicated whether $K=K^{*}, K=K^{\dagger}, K^{*}=K^{\dagger}, K=-K$ or not. In the following we use Green's notation for oriented virtual knots with up to 4 crossings.

2.3. Welded virtual knot. There are also the so-called forbidden moves $\Phi_{t}, \Phi_{h}$ as shown in Fig. 6. It is known $[12,24]$ that if we allow both of these moves, any virtual knot becomes a trivial knot.
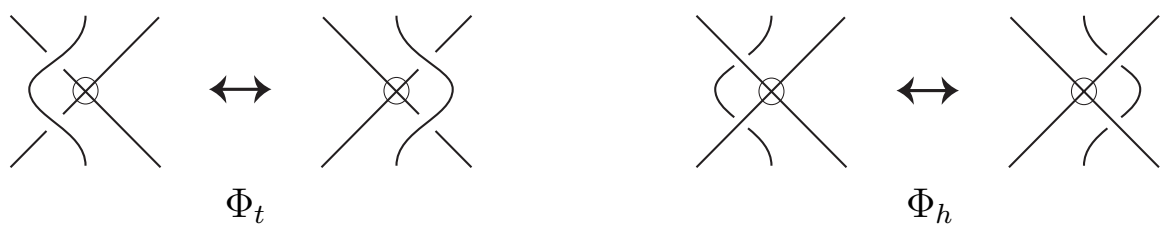

FiguRE 6. Forbidden moves.

A welded virtual knot $[2,26]$ is an equivalence class of virtual knot diagrams under the generalized Reidemeister moves, as well as one of the forbidden moves $\Phi_{t}$. Let $K$, $K^{\prime}$ be oriented virtual knots with diagrams $D, D^{\prime}$, respectively. Then $K$ and $K^{\prime}$ are oriented welded-equivalent, denoted by $K \stackrel{\text { ow }}{\sim} K^{\prime}$, if the diagrams $D$ and $D^{\prime}$ are related by a finite sequence of generalized Reidemeister moves and the move $\Phi_{t}$. There are moves of Gauss diagrams corresponding to the forbidden moves $\Phi_{t}, \Phi_{h}$; see [24, Figure 4]. In Fig. 7 we give the move corresponding to $\Phi_{t}$, which moves an arrowtail of either sign past an adjacent arrowtail with either sign without conditions on the relative positions of the heads of these arrows. We also denote this move on Gauss diagrams by the same symbol $\Phi_{t}$. Thus if Gauss diagrams $\operatorname{Gau} \beta(D)$ and $\operatorname{Gau}\left(D^{\prime}\right)$ are related by a finite sequence of the moves $\Omega_{1}, \Omega_{2}, \Omega_{3}, \Phi_{t}$, then $K \stackrel{\text { ow }}{\sim} K^{\prime}$. 


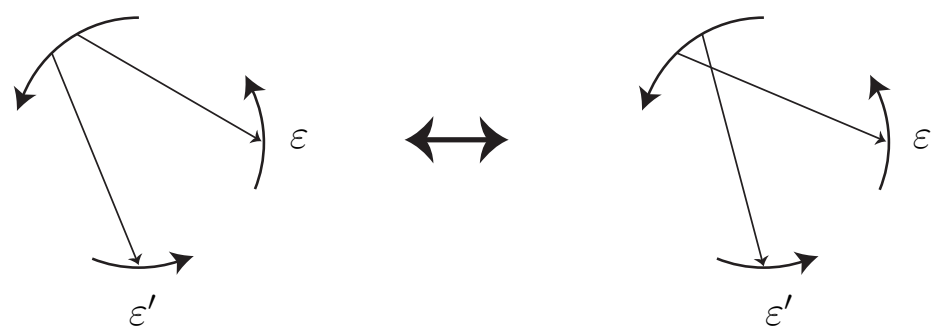

Figure 7. The move of Gauss diagrams corresponding to the forbidden move $\Phi_{t}$, where $\varepsilon, \varepsilon^{\prime}=+$ or - .

Example 2.4. The knot 3.1 given in Fig. 3 is oriented welded-equivalent to the trivial knot. In fact, using the moves $\Phi_{t}$ and $\Omega_{1}$, we may deform the Gauss diagram of 3.1 given in Fig. 5 into that of the trivial knot as shown in Fig. 8.

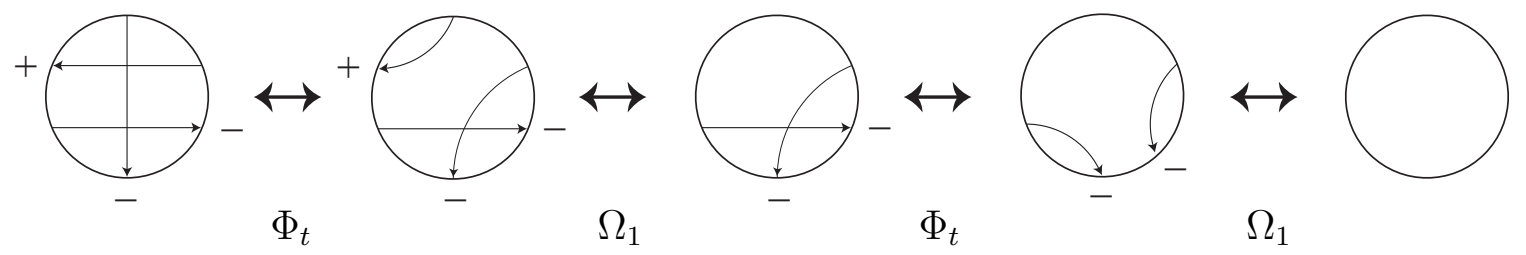

Figure 8. Deformation of the Gauss diagram of the knot 3.1.

2.4. Group of a virtual knot. For a virtual knot diagram $D$ of an oriented virtual knot $K$, the group of $D, G(D)$, is given by a generalization of Wirtinger's algorithm $[15,16]$ : one associates a generator to each arc in the diagram. The relations are determined by the classical crossings as shown in Fig. 9. The group of a virtual knot is invariant under all the generalized Reidemeister moves and also the forbidden move $\Phi_{t}$, and hence is an invariant of welded virtual knots, and so we also denote by $G(K)$ the group of the (welded) virtual knot $K$ presented by the diagram $D$.
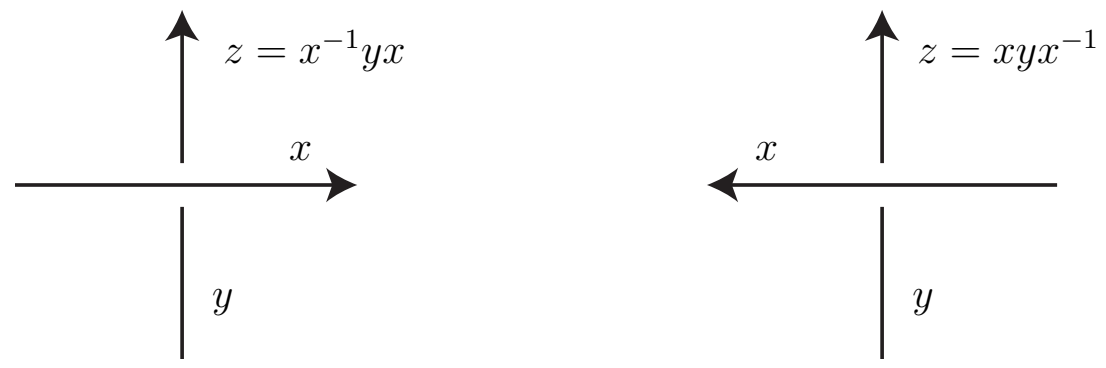

Figure 9. Generators and relations for the group of a virtual knot. 

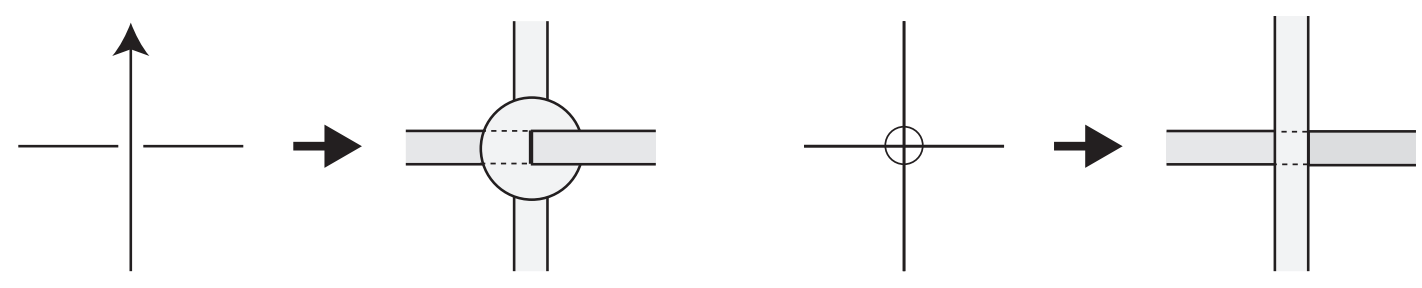

FIGURE 10. Virtual knot presentation.

\section{ViRTUAL KNOt PRESENTATiON OF A RIBBON TORUS KNOT}

A surface knot is an embedded surface in the 4 -space $\mathbf{R}^{4}$. In this paper, we consider either a sphere knot (i.e., a 2-knot) or a torus knot (or a $T^{2}$ knot). For an oriented surface knot $F$, we denote by $-F$ and $F^{*}$ the surface knots obtained by reversing the orientation and taking the mirror image, respectively. Two oriented surface knots $F$ and $F^{\prime}$ are oriented equivalent, denoted by $F \stackrel{ }{\approx} F^{\prime}$, if there exists an ambient isotopy of $\mathbf{R}^{4}$ which maps $F$ to $F^{\prime}$ preserving the orientation, and equivalent, denoted by $F \approx F^{\prime}$, if $F \stackrel{ }{\approx} F^{\prime}$ or $-F^{\prime}$.

A ribbon surface knot is an embedded surface in $\mathbf{R}^{4}$ obtained by adding $q$ 1-handles to a trivial 2-link with $p$ components for some $p$ and $q$. Thus for a ribbon 2-knot $p=q+1$ and for a ribbon torus knot $p=q$; cf. [18, p. 178].

A ribbon handlebody is a 2-dimensional handlebody in $\mathbf{R}^{3}$ consisting of $p$ 0-handles $\Delta=D_{1} \cup \cdots \cup D_{p}$ and $q$ 1-handles $B=B_{1} \cup \cdots \cup B_{q}$ for some $p$ and $q$, which has only ribbon singularities; the preimage of each ribbon singularity consists of an arc in the interior of a 0-handle and a cocore of a 1-handle. In order to present a ribbon handlebody we use a ribbon regular projection as in [10, Sect. 1] or [11, Sect. 3]. We define the associated ribbon surface knot in $\mathbf{R}^{4}=\mathbf{R}^{3} \times \mathbf{R}$ as the ribbon surface knot that bounds the immersed 3-disk $\Delta \times[-2,2] \cup B \times[-1,1]$. Conversely, for any ribbon surface knot $K$, there exists a ribbon handlebody whose associated ribbon surface knot is $K$; see $[13,32]$. Thus we may represent a ribbon surface knot by a ribbon regular projection of a ribbon handlebody.

Given an oriented virtual knot diagram of a virtual knot $K$, we can construct a ribbon regular projection of a ribbon handlebody as shown in Fig. 10; note that a classical crossing corresponds to a 0-handle. From this we obtain an associated ribbon torus knot, which we denote by Tube $(K)$. Satoh has shown [26, Proposition 3.3] that an oriented welded virtual knot determines a ribbon torus knot by this operation. Conversely, he has shown [26, Theorem 3.1] that for any ribbon torus knot $T$, there exists an oriented virtual knot $K$ such that $\operatorname{Tube}(K)=T$. Then the group of the virtual knot $K$ is isomorphic to the fundamental group of the complement of Tube $(K)$; $G(K) \cong \pi_{1}\left(\mathbf{R}^{4}-\right.$ Tube $\left.(K)\right)$ [26, Proposition 3.4]. For a surface knot $F$ in $\mathbf{R}^{4}$ we also denote by $G(F)$ the fundamental group $\pi_{1}\left(\mathbf{R}^{4}-F\right)$, and thus $G(K) \cong G($ Tube $(K))$. 
Example 3.1. From the diagram of the knot 3.1 given in the first diagram in Fig. 3 we obtain the ribbon regular projection of a ribbon handlebody as shown in Fig. 11, which consists of three 0-handles $D_{1} \cup D_{2} \cup D_{3}$ and three 1-handles $B_{1} \cup B_{2} \cup B_{3}$; each 0 -handle $D_{i}$ corresponds to the real crossing assigned as $i, i=1,2,3$, in the first diagram in Fig. 11, and the 1-handle $B_{i}$ connects the two 0-handles $D_{i-1}$ and $D_{i}$, where the subscripts are taken modulo 3.

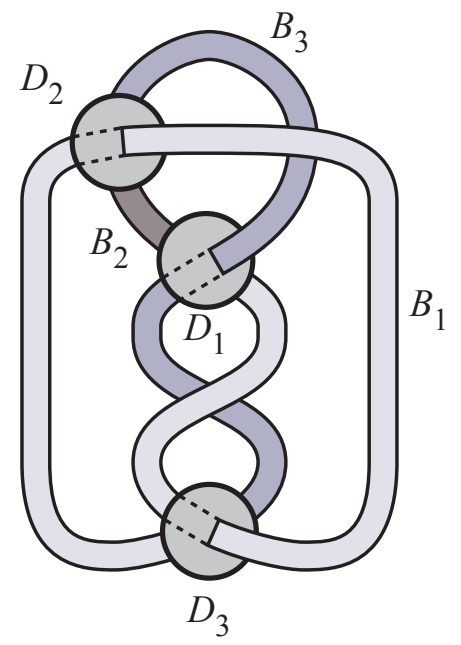

FiguRE 11. Ribbon handlebody constructed from the knot 3.1.

For a ribbon surface knot we have the following property due to Yoshihiko Marumoto; see [29, Theorem 2.18], [26, Proposition 4.1].

Proposition 3.2. A ribbon surface knot is (-)amphicheiral, that is, if $F$ is a ribbon surface knot, then

$$
F \stackrel{\circ}{\approx}-F^{*}
$$

However, it is not always the case that a ribbon surface knot is reversible, $F \stackrel{0}{\approx}-F$, or equivalently $(+)$ amphicheiral, $F \stackrel{\mathrm{O}}{\approx} F^{*}$.

Definition 1. A ribbon torus knot $F$ is symmetric if it is reversible or $(+)$ amphicheiral; $F \stackrel{\circ}{\approx}-F \stackrel{\mathrm{o}}{\approx} F^{*}$, and asymmetric otherwise.

For an oriented virtual knot $K$, Satoh [26, Corollary 4.2] has shown:

$$
\operatorname{Tube}(-K) \stackrel{\mathrm{o}}{\approx}-\operatorname{Tube}(K),
$$

and Winter [30, Theorem 3.6] has shown:

$$
\operatorname{Tube}(K)^{*} \stackrel{\mathrm{o}}{\approx} \operatorname{Tube}\left(-K^{\dagger}\right)
$$


As we have seen in Example 2.1, in general eight oriented virtual knots $K, K^{*}, K^{\dagger}$, $K^{* \dagger},-K,-K^{*},-K^{\dagger},-K^{* \dagger}$ are mutually distinct. However, using Eqs. (3.1)-(3.3), we obtain the following; cf. [30, 31].

Proposition 3.3. For an oriented virtual knot $K$,

$$
\begin{aligned}
& \operatorname{Tube}\left(K^{\dagger}\right) \stackrel{\mathrm{o}}{\approx} \operatorname{Tube}(K) ; \\
& \operatorname{Tube}(-K) \stackrel{\mathrm{o}}{\approx} \operatorname{Tube}\left(-K^{\dagger}\right) \stackrel{\mathrm{o}}{\approx}-\operatorname{Tube}(K) ; \\
& \operatorname{Tube}\left(K^{* \dagger}\right) \stackrel{\mathrm{o}}{\approx} \operatorname{Tube}\left(K^{*}\right) \text {; } \\
& \operatorname{Tube}\left(-K^{*}\right) \stackrel{\mathrm{o}}{\approx} \operatorname{Tube}\left(-K^{* \dagger}\right) \stackrel{\mathrm{o}}{\approx}-\operatorname{Tube}\left(K^{*}\right),
\end{aligned}
$$

and thus, we have

$$
\begin{aligned}
\operatorname{Tube}(K) & \approx \operatorname{Tube}\left(K^{\dagger}\right) \approx \operatorname{Tube}(-K) \approx \operatorname{Tube}\left(-K^{\dagger}\right) \\
\operatorname{Tube}\left(K^{*}\right) & \approx \operatorname{Tube}\left(K^{* \dagger}\right) \approx \operatorname{Tube}\left(-K^{*}\right) \approx \operatorname{Tube}\left(-K^{* \dagger}\right) .
\end{aligned}
$$

This proposition immediately implies the following:

Proposition 3.4. Suppose that $\Gamma_{1}$ and $\Gamma_{2}$ are the Gauss diagrams of diagrams for oriented virtual knots $K_{1}$ and $K_{2}$, respectively. Then:

(i) If either $\Gamma_{1} \stackrel{\text { ow }}{\sim} \Gamma_{2}$ or $\Gamma_{1} \stackrel{\text { ow }}{\sim} \Gamma_{2}^{\dagger}$, then $\operatorname{Tube}\left(K_{1}\right) \stackrel{\mathrm{o}}{\sim} \operatorname{Tube}\left(K_{2}\right)$.

(ii) If either $\Gamma_{1} \stackrel{\text { ow }}{\sim}-\Gamma_{2}$ or $\Gamma_{1} \stackrel{\text { ow }}{\sim}-\Gamma_{2}^{\dagger}$, then $\operatorname{Tube}\left(K_{1}\right) \stackrel{\text { o }}{\approx}-\operatorname{Tube}\left(K_{2}\right)$.

In particular, if an oriented virtual knot $K$ is reversible, or equivalently the Gauss diagram $\Gamma$ for $K$ satisfies $\Gamma=-\Gamma$, then $\operatorname{Tube}(K)$ is symmetric; $\operatorname{Tube}(K) \stackrel{\mathrm{o}}{\approx}-\operatorname{Tube}(K)$.

Let $F$ be a torus knot in $\mathbf{R}^{4}$ and $X$ its exterior. The peripheral subgroup of $F$ is the image of $\pi_{1}(\partial X)$ by the map $i_{*}$ induced by the inclusion $i: \partial X \rightarrow X$. So it is isomorphic to the direct sum of $\mathbf{Z}$, which is generated by a meridian, and some quotient of $\pi_{1}(F) \cong \mathbf{Z} \oplus \mathbf{Z}$. If $F$ is a ribbon torus knot given by Tube $(K)$ for some virtual knot $K$, then the peripheral subgroup is generated by the meridian and the longitude, which is obtained as an element of the knot group of $K$ corresponding to circling the knot exactly once in the direction of the orientation of the knot without algebraic linking; cf. $[4,19,30]$.

\section{Statement of the Result}

The first author [9] has calculated the groups of virtual knots with up to 4 crossings in Green's table, and has shown that each of them is isomorphic to either the infinite cyclic group or one of the following 9 groups. The first author showed that they are 
mutually distinct by using the elementary ideals.

$$
\begin{aligned}
& G_{1}=\langle x, y \mid x y x=y x y\rangle, \\
& G_{2}=\left\langle x, y \mid x y x=y x y, y=x^{-2} y x^{2}\right\rangle, \\
& G_{3}=\left\langle x, y \mid x y x=y x y, y=x^{-3} y x^{3}\right\rangle, \\
& G_{4}=\left\langle x, y \mid x^{-1} y x y^{-1} x=y x^{-1} y x y^{-1}\right\rangle, \\
& G_{5}=\left\langle x, a \mid x^{-1} a x=a^{-1}, a^{5}=1\right\rangle, \\
& G_{6}=\left\langle x, a \mid x^{-1} a x=a^{2}, a^{5}=1\right\rangle, \\
& G_{7}=\left\langle x, y \mid x^{-1} y x=y^{-1} x y\right\rangle, \\
& G_{8}=\left\langle x, a \mid x^{-1} a x=a^{2}, a^{7}=1\right\rangle, \\
& G_{9}=\left\langle x, y \mid x y x y^{-1} x^{-1}=y x^{-1} y x y^{-1}, x^{-1} y^{-1} x y x=y^{-1} x^{-1} y x y\right\rangle .
\end{aligned}
$$

The group $G_{1}$ is the trefoil knot group, and $G_{2}$ and $G_{3}$ are the groups of the 2- and 3 -twist spun trefoil knot, respectively; see [34]. The group $G_{4}$ is the figure-eight knot group, and $G_{5}$ is the group of the 2-twist spun of either the figure-eight knot or $(2,5)$ torus knot. The group $G_{7}$ is the group of a ribbon 2-knot given by Fox [3, Example 11]. The commutator subgroups of $G_{6}$ and $G_{8}$ are cyclic of order 5 and 7 , respectively, which have been given as the groups of some ribbon torus knots in $[1,7]$; it is known that these groups are of some 3 -knots but not of any 2 -knots (Remarks 9.2, 12.3). We prove that the commutator subgroup of $G_{9}$ is abelian (Proposition 10.2), and using a result of Hillman, we can see it is not a 2-knot group (Remark 10.3).

Our main result is the following:

Theorem 4.1. Let $K$ be a virtual knot with up to 4 classical crossings. If the group of $K$ is not infinite cyclic, then the corresponding ribbon torus knot Tube $(K)$ is equivalent to one of the 13 knots listed in Table 1, which are mutually inequivalent except for the two paris $\left\{\mathrm{T}_{8}, \mathrm{~T}_{4}\right\}$ and $\left\{\mathrm{T} 4_{10}, \mathrm{~T}_{11}\right\}$. (We do not know whether these pairs are equivalent or not.)

Proof. The first author [9] has calculated the groups of the virtual knots with up to 4 crossings. So, for each group $G_{i}$ we will examine the ribbon torus knots represented by virtual knots with up to 4 crossings with group $G_{i}$ in Secs. 5-12 to obtain Table 1.

Table 1 summarizes our result: We define 13 ribbon torus knots $\mathrm{T} 3_{1}, \mathrm{~T} 3_{2}, \mathrm{~T} 4_{i}$, $i=1,2, \ldots, 11$, obtained from the virtual knots with up to 4 crossings through the Tube operation as in the first and second columns in Table 1, where the Gauss diagrams for the virtual knots in the second column are shown in Fig. 12. The third column in Table 1 indicates the symmetry of the ribbon torus knots, where the symbols "s", "a", "?" mean that it is symmetric, asymmetric, or unknown whether symmetric or not, respectively. In the last column we give other virtual knots representing the ribbon torus knots. So the groups of the remaining virtual knots are infinite cyclic. 
TABLE 1. Ribbon torus knots represented by virtual knots with up to 4 crossings.

\begin{tabular}{c|l|c|c|l}
\hline Tube $(K)$ & $K$ & Group & Symmetry & Other knots representing Tube $(K)$ \\
\hline $\mathrm{T} 3_{1}$ & $3.6\left(\sim 3.6^{* \dagger}\right)$ & $G_{1}$ & $\mathrm{~s}$ & $4.61,4.68,4.69^{*}, 4.73^{*}$. \\
$\mathrm{T} 3_{2}$ & $3.7\left(\sim 3.7^{* \dagger}\right)$ & $G_{2}$ & $\mathrm{~s}$ & $4.62-4.67,4.98,4.70^{*}, 4.74^{*}, 4.76^{*}$. \\
$\mathrm{T} 4_{1}$ & $4.108\left(\sim 4.108^{*}\right)$ & $G_{4}$ & $\mathrm{~s}$ & \\
$\mathrm{~T} 4_{2}$ & $4.71^{*}$ & $G_{1}$ & $\mathrm{~s}$ & $4.75^{*}, 4.98^{*}$. \\
$\mathrm{T} 4_{3}$ & $4.65^{*}$ & $G_{3}$ & $\mathrm{~s}$ & 4.96. \\
$\mathrm{~T} 4_{4}$ & $4.102\left(\sim 4.102^{* \dagger}\right)$ & $G_{3}$ & $\mathrm{~s}$ & $4.104\left(\sim 4.104^{* \dagger}\right)$. \\
$\mathrm{T} 4_{5}$ & $4.99\left(\sim 4.99^{*}\right)$ & $G_{7}$ & $\mathrm{a}$ & $4.72^{*}, 4.77^{*}$. \\
$\mathrm{T} 4_{6}$ & $4.62^{*}$ & $G_{8}$ & $\mathrm{a}$ & $4.95,4.95^{*}$. \\
$\mathrm{T} 4_{7}$ & 4.105 & $G_{9}$ & $\mathrm{a}$ & $4.105^{*}$. \\
$\mathrm{T} 4_{8}$ & $4.106\left(\sim 4.106^{* \dagger}\right)$ & $G_{5}$ & $\mathrm{~s}$ & \\
$\mathrm{~T} 4_{9}$ & 4.107 & $G_{5}$ & $?$ & $4.107^{*}$. \\
$\mathrm{T}_{10}$ & $4.66^{*}$ & $G_{6}$ & $\mathrm{a}$ & 4.97. \\
$\mathrm{~T} 4_{11}$ & $4.101^{*}\left(\sim 4.101^{\dagger}\right)$ & $G_{6}$ & $\mathrm{a}$ & $4.103\left(\sim 4.103^{* \dagger}\right)$. \\
\hline
\end{tabular}

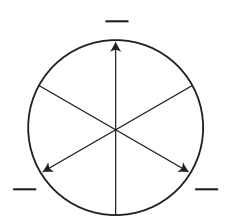

3.6

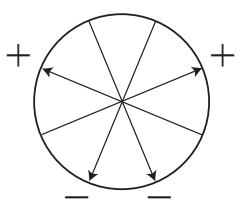

4.102

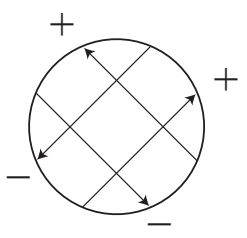

4.107

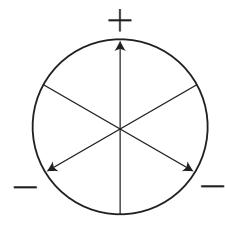

3.7

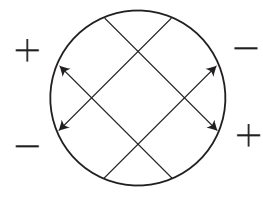

4.99

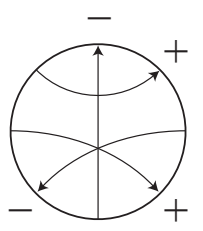

$4.66^{*}$

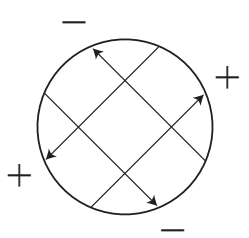

4.108

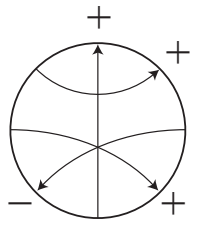

$4.62^{*}$

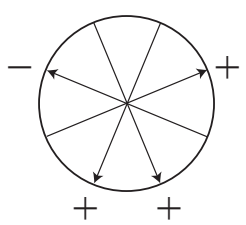

$4.101^{*}$

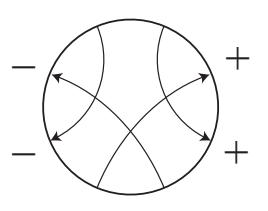

$3.71^{*}$

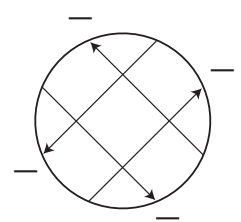

4.105

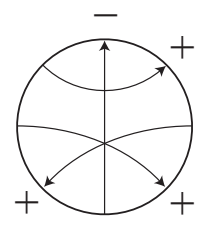

$4.65^{*}$

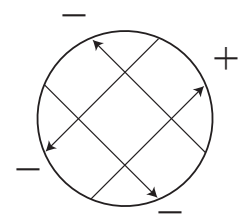

4.106

FiguRE 12. Gauss diagrams for the virtual knots defining ribbon torus knots in Table 1. 
5. RibBon torus knots with groups $G_{1}$ OR $G_{4} ; \mathrm{T} 3_{1}, \mathrm{~T} 4_{1}, \mathrm{~T} 4_{2}$

The groups $G_{1}, G_{4}$ are the trefoil knot group, figure-eight knot group, respectively. Given a classical knot $K$, we can construct ribbon torus knots whose groups are isomorphic to that of $K$ in two ways; one is the spun torus knot, and the other is adding a trivial 1-handle to the spun 2-knot of $K$. These ribbon torus knots are inequivalent because they have different peripheral subgroups; see [26, Page 541]. In Subsec. 5.1, we consider $\mathrm{T} 3_{1}$ and $\mathrm{T} 4_{1}$, which are spun torus knots of the trefoil and figure-eight knots, respectively. In Subsec. 5.2, we consider the ribbon torus knot $\mathrm{T}_{2}$, which is obtained from the spun trefoil knot by adding a trivial 1-handle.

5.1. Spun torus knots; $\mathrm{T} 3_{1}, \mathrm{~T} 4_{1}$. A spun torus knot of a classical knot $K$ is defined as follows: Put $K$ in the half 3 -space $\mathbf{R}_{+}^{3}$, and then rotate $\mathbf{R}_{+}^{3}$ about $\mathbf{R}^{2}$, we obtain the spun torus knot of $K$ in the 4-space. Satoh [26, Theorem 4.3] has shown that if $K$ is a classical knot, then Tube $(K)$ is a spun torus knot of $K$. So the group of the spun torus knot of $K$ is isomorphic to that of $K$; cf. [22, Proposition 3.1(a)]. We define $\mathrm{T} 3_{1}=$ Tube(3.6) and $\mathrm{T} 4_{1}=$ Tube(4.108), where the knots 3.6, 4.108 are the left-hand trefoil knot and figure-eight knot, respectively. Then we have the following.

Lemma 5.1. (i) The ribbon torus knots $\mathrm{T} 3_{1}$ and $\mathrm{T} 4_{1}$ are symmetric.

(ii) $G\left(\mathrm{~T} 3_{1}\right) \cong G(3.6) \cong G_{1} ; G\left(\mathrm{~T} 4_{1}\right) \cong G(4.108) \cong G_{4}$.

(iii) For $K=3.6^{*}, 4.61,4.68,4.69^{*}, 4.73^{*}$, $\operatorname{Tube}(K) \stackrel{\mathrm{o}}{\approx} \mathrm{T} 3_{1}$.

Proof. (i) Since the trefoil knot 3.6 and figure-eight knot 4.108 are reversible, T3 1 and $\mathrm{T} 4_{1}$ are symmetric by Proposition 3.4. The claim (ii) is obvious.

(iii) From the Gauss diagrams as shown in Figs. 12 and 13 , we have $3.6 \stackrel{\text { ow }}{\sim} 4.61$, $3.6^{*} \stackrel{\text { ow }}{\sim} 4.68$, and $4.69^{*} \stackrel{\text { ow }}{\sim} 4.73^{*}$. Figure 14 shows that $4.69^{*} \stackrel{\text { ow }}{\sim} 3.6^{*}$. Since $3.6^{*}=3.6^{\dagger}$, by Eq. (3.4) we have the result. This completes the proof.

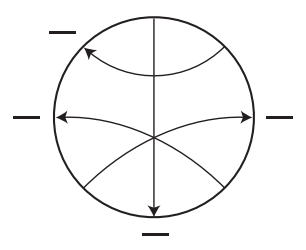

4.61

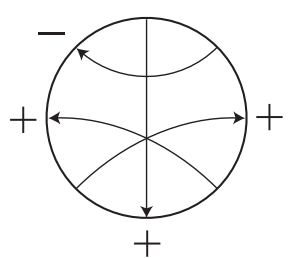

4.68

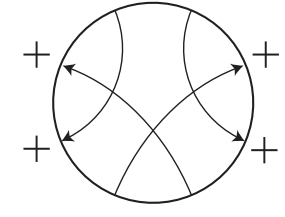

$4.69^{*}$

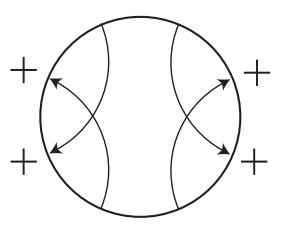

$4.73^{*}$

Figure 13. Gauss diagrams for virtual knots representing $\mathrm{T} 3_{1}$.

5.2. Ribbon torus knot $\mathrm{T} 4_{2}$. We define $\mathrm{T} 4_{2}=\operatorname{Tube}\left(4.71^{*}\right)$.

Lemma 5.2. (i) The ribbon torus knot $\mathrm{T}_{2}$ is obtained from the spun trefoil knot by adding a trivial 1-handle.

(ii) $G\left(\mathrm{~T} 4_{2}\right) \cong G\left(4.71^{*}\right) \cong G_{1}$. 


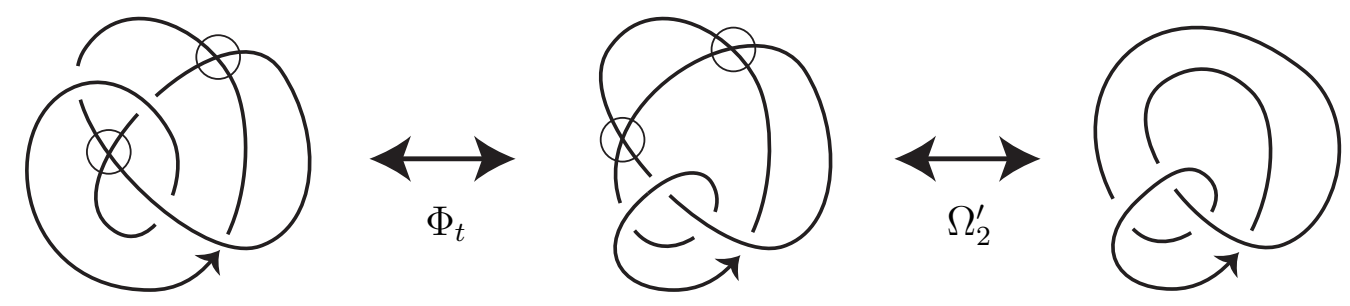

4.69*

Figure 14 . The knot $4.69^{*}$ is oriented welded-equivalent to the righthand trefoil knot $3.6^{*}$.

(iii) For $K=4.75^{*}, 4.98^{*}, \operatorname{Tube}(K) \stackrel{\mathrm{o}}{\approx} \mathrm{T}_{2}$.

(iv) $\mathrm{T}_{2}$ is symmetric.

Proof. In Example 5.10 in [26] it is shown that a ribbon torus knot obtained from the spun trefoil knot by adding a trivial 1-handle is represented by a virtual knot diagram as shown in Fig. 15; cf. [17, Sec. 3.4]. Its Gauss diagram is the same as that of $4.71^{*}$ (Fig. 12), and so we obtain (i). The claim (ii) is also remarked there.

From the Gauss diagrams in Figs. 12 and 16 , we have $4.71^{*} \stackrel{\text { ow }}{\sim} 4.75^{*} \stackrel{\text { ow }}{\sim} 4.98^{*}$, giving (iii). Since $-\operatorname{Gau}\left(4.75^{*}\right)=\operatorname{Gau}\left(4.75^{*}\right)$, by Proposition 3.4 we have (iv). This completes the proof.

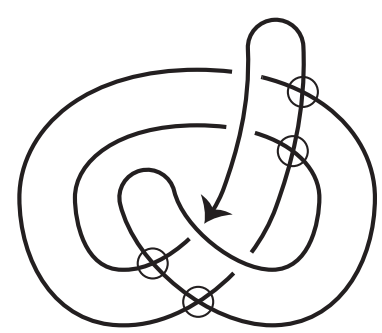

Figure 15. A diagram of the knot $4.71^{*}$.

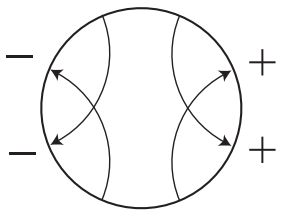

$4.75^{*}$

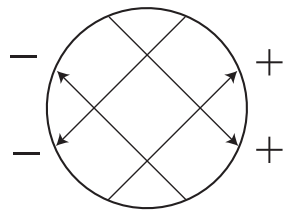

$4.98^{*}$

FiguRE 16. Gauss diagrams of virtual knots representing $\mathrm{T} 4_{2}$. 


\section{RibBon torus KNOT With GROUP $G_{2} ; \mathrm{T} 3_{2}$}

We difine $\mathrm{T} 3_{2}=$ Tube(3.7). Note that the knot 3.7 is the 2-braid virtual knot $B(-2,1)$ as shown in Fig. 17 .

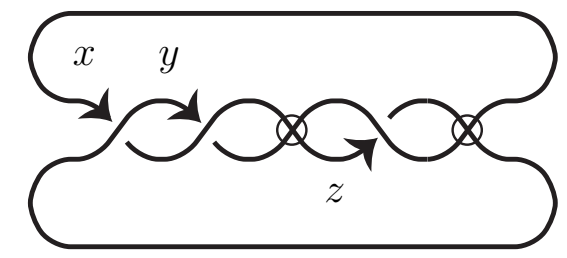

Figure 17. The knot 3.7 is the virtual 2-braid knot $B(-2,1)$.

Lemma 6.1. (i) The ribbon torus knot $\mathrm{T} 3_{2}$ is symmetric.

(ii) $G\left(\mathrm{~T} 3_{2}\right) \cong G(3.7) \cong G_{2}$.

(iii) For $K=3.7^{*}, 4.62-4.67,4.70^{*}, 4.74^{*}, 4.76^{*}, 4.98$, $\operatorname{Tube}(K) \stackrel{\mathrm{o}}{\approx} \mathrm{T} 3_{2}$.

Proof. (i) Green [5] mentions that the knot 3.7 is reversible, which implies the result. In fact, from the diagram shown in Fig. 17 it is easy to see that the knot 3.7 is reversible. This also follows from $\operatorname{Gau} \beta(3.7)=-\operatorname{Gauß}(3.7)$.

(ii) Using the Wirtinger generators $x, y, z$ in Fig. 17, we have

$$
G(3.7)=\langle x, y, z, \mid z y=y x, x z=z y, x y=z x\rangle .
$$

Removing $z$, we obtain the presentation $G_{2}$. Putting $a=y x^{-1}$, we have

$$
G_{2} \cong\left\langle x, a \mid x^{-1} a x=a^{-1}, a^{3}=1\right\rangle
$$

showing the commutator subgroup is isomorphic to the cyclic group of order 3 ; see [28, Example 1.1], [14, Sec. 6].

(iii) We give the Gauss diagrams for the the knots $3.7,3.7^{*}$, and $4.62, \ldots, 4.67$ in Fig. 18, where the third diagram gives the Gauss diagrams for the knots $4.62, \ldots, 4.67$ with signs indicated in Table 2. Since $\operatorname{Gau} \beta\left(3.7^{*}\right)=\operatorname{Gauß}(3.7)^{\dagger}$, by Proposition 3.4 we have Tube $\left(3.7^{*}\right) \stackrel{\mathrm{o}}{\approx} \mathrm{T} 3_{2}=\operatorname{Tube}(3.7)$. Indeed, Green [5] mentions that $3.7^{*} \sim 3.7^{\dagger}$.

The third Gauss diagram in Fig. 18 is oriented welded-equivalent to the fourth one, which is the Gauss diagram for either 3.7 or $3.7^{*}$, and so $\operatorname{Tube}(K) \stackrel{\mathrm{o}}{\approx} \mathrm{T} 3_{2}$ for $K=4.62$, $\ldots, 4.67$.

Now we consider the remaining knots. In Fig. 19 we give the Gauss diagrams for the knots $4.70^{*}, 4.74^{*}, 4.76^{*}, 4.98^{*}$, which implies $4.70^{*} \stackrel{\text { ow }}{\sim}-4.74^{*}, 4.76^{*} \stackrel{\text { ow }}{\sim} 4.98^{*}$, and so Tube $\left(4.70^{*}\right) \stackrel{\mathrm{o}}{\approx}-\operatorname{Tube}\left(4.74^{*}\right)$, Tube $\left(4.76^{*}\right) \stackrel{\mathrm{o}}{\approx} \operatorname{Tube}\left(4.98^{*}\right)$.

Then, we show that $4.74^{*}$ and $4.76^{*}$ are oriented welded-equivalent to 3.7 . The moves as shown in Fig. 20 are realized by the generalized Reidemeister moves. Applying them to the diagram of the knot 3.7 in Fig. 21(a), we have two diagrams Figs. 21(b) and (c), both of which are oriented equivalent to the first diagram of 3.7. The Gauss diagrams 


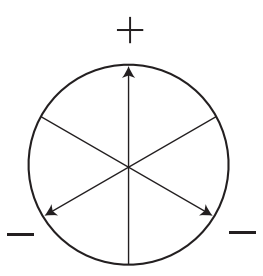

3.7

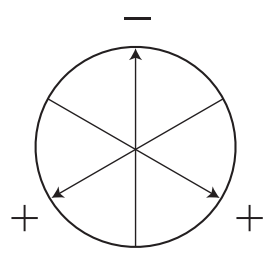

$3.7^{*}$

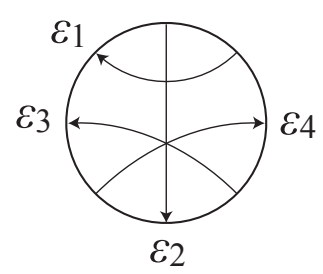

$4.62-4.67$

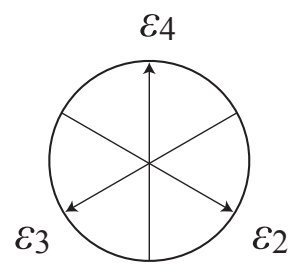

, 4.67 .

4.67.

\begin{tabular}{c|cccc}
\hline knot & $\varepsilon_{1}$ & $\varepsilon_{2}$ & $\varepsilon_{3}$ & $\varepsilon_{4}$ \\
\hline 4.62 & - & - & - & + \\
4.63 & - & - & + & - \\
4.64 & - & - & + & + \\
4.65 & - & + & - & - \\
4.66 & - & + & - & + \\
4.67 & + & + & - & - \\
\hline
\end{tabular}

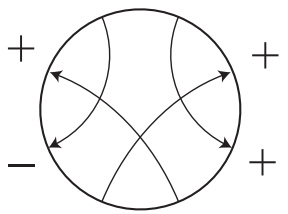

$4.70^{*}$

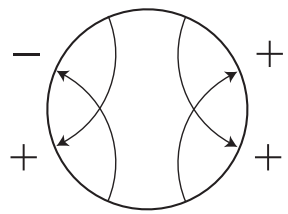

$4.74^{*}$

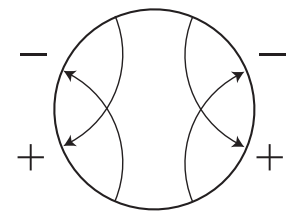

$4.76^{*}$

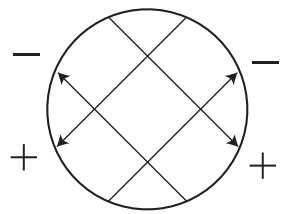

4.98

FiguRE 19. Gauss diagram for the knots $4.70^{*}, 4.74^{*}, 4.76^{*}, 4.98$.

for them are given in Fig. 22; the first one is oriented welded-equivalent to the Gauss diagram $\Gamma^{\dagger}$, where $\Gamma$ is the Gauss diagram for $4.74^{*}$ given in Fig. 19, and the second one is oriented welded-equivalent to the Gauss diagram for $4.76^{*}$ given in Fig. 19. This completes the proof.

7. Ribbon torus KnOts With group $G_{3} ; \mathrm{T}_{3}, \mathrm{~T}_{4}$

There are two ribbon torus knots with group $G_{3}$ represented by virtual knots with up to 4 crossings; $\mathrm{T}_{3}, \mathrm{~T}_{4}$.

7.1. Ribbon torus knot $\mathrm{T} 4_{3}$. We define $\mathrm{T} 4_{3}=$ Tube $\left(4.65^{*}\right)$.

Lemma 7.1. (i) The ribbon torus knot $\mathrm{T}_{3}$ is symmetric.

(ii) $G\left(\mathrm{~T} 4_{3}\right) \cong G\left(4.65^{*}\right) \cong G_{3}$. 

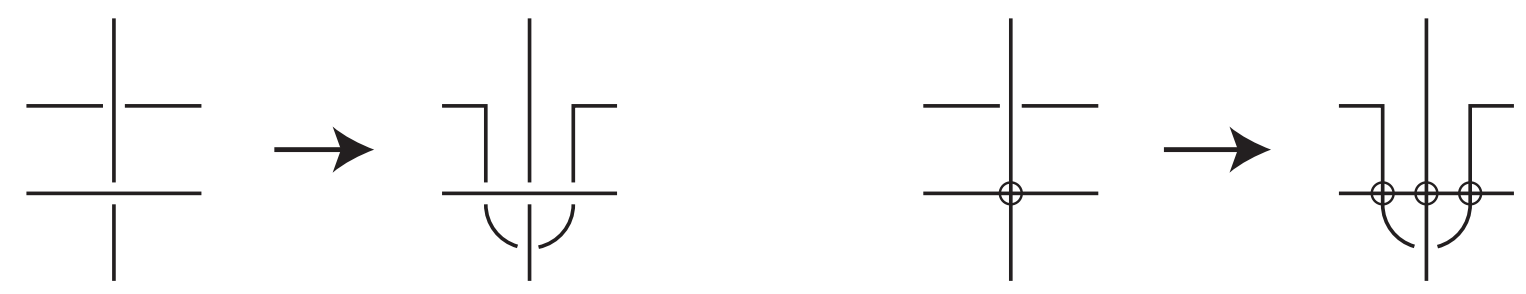

FiguRE 20. Moves realized by a finite sequence of generalized Reidemeister moves.

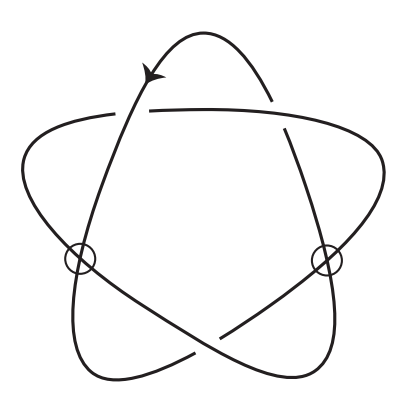

(a)

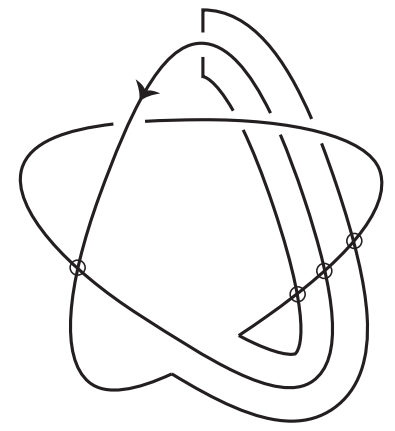

(b)

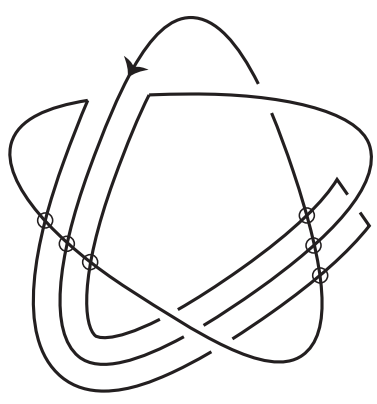

(c)

Figure 21. Three diagrams of the knot 3.7.
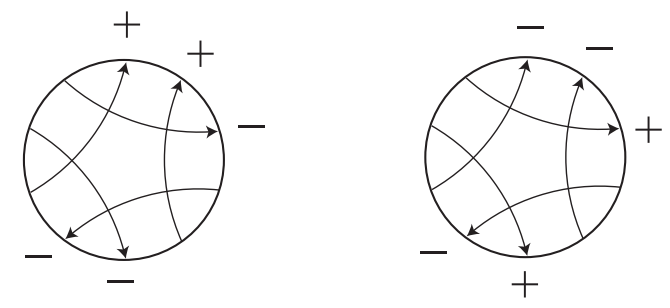

Figure 22. Gauss diagrams for the knot diagrams in Figs. 21(b), (c).

(iii) The longitude of $\mathrm{T}_{3}$ is the unit element in $G\left(\mathrm{~T}_{3}\right)$.

(iv) $\operatorname{Tube}(4.96) \stackrel{\mathrm{o}}{\approx} \mathrm{T}_{3}$.

Proof. (i) Green [5] mentions that the virtual knot 4.65 is reversible, and so is $4.65^{*}$, which implies that $\mathrm{T}_{3}$ is symmetric. In fact, in Fig. 23 a sequence of deformations of the knot $4.65^{*}$ into a diagram which indicates the reversibility of $4.65^{*}$; rotating the whole diagram 180 degrees, we obtain the reversion. 


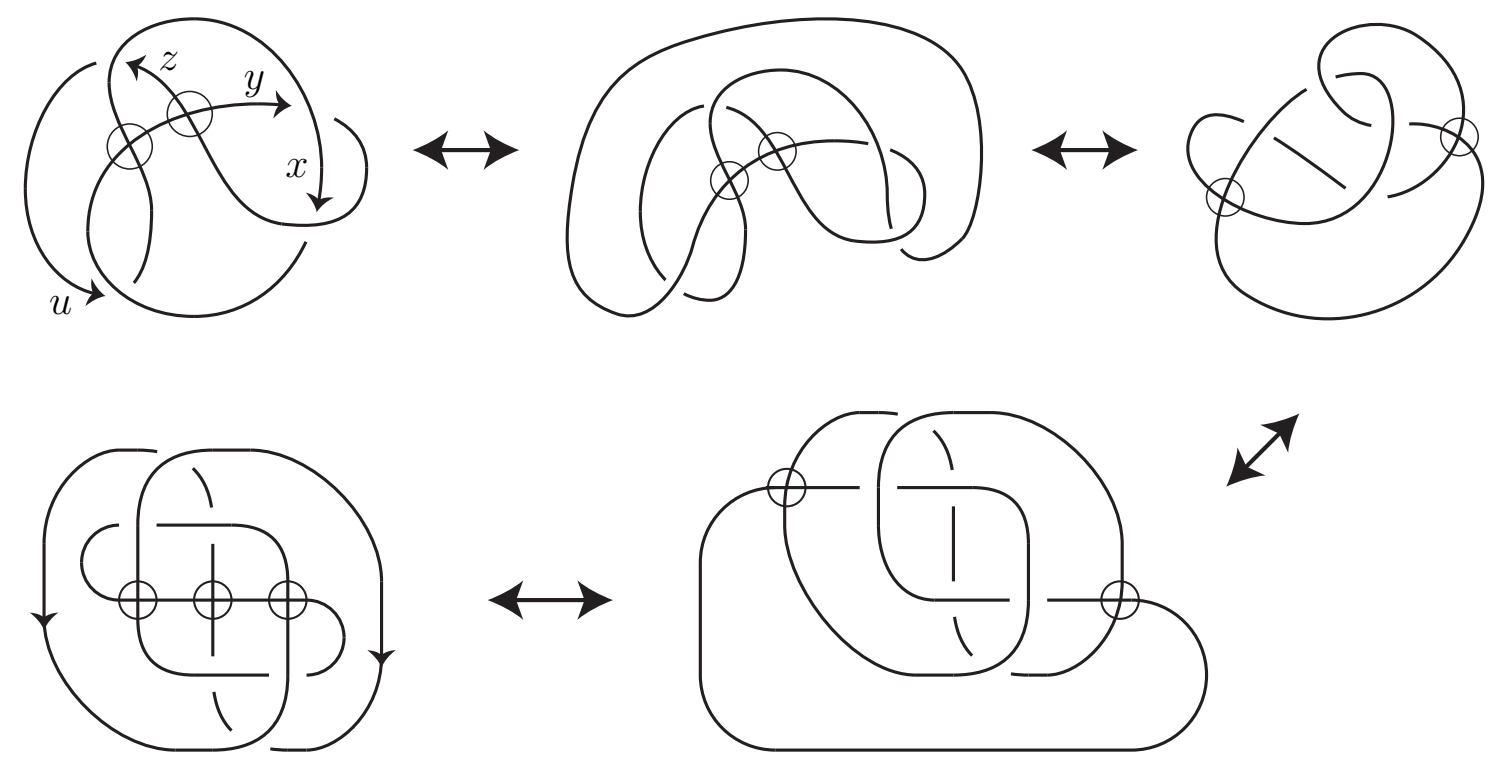

Figure 23. A sequence of a deformation of the knot $4.65^{*}$.

(ii) Using the generators given in the first diagram in Fig. 23, we have:

$$
\begin{aligned}
G\left(4.65^{*}\right) & =\langle x, y, z, u \mid y x=x z=z y, y u=x y, x u=z x\rangle \\
& \cong\left\langle x, y, z \mid y x=x z=z y, y^{-1} x y=x^{-1} z x\right\rangle \\
& \cong\left\langle x, y \mid y x=x^{2} y^{-1} x y x^{-1}=x y^{-1} x y x^{-1} y\right\rangle,
\end{aligned}
$$

where we eliminate the generators $u$ and $z$. From the relations $y x=x^{2}\left(y^{-1} x y\right) x^{-1}$ and $y x=x\left(y^{-1} x y\right) x^{-1} y$, we obtain

$$
y^{-1} x y=x^{-2} y x^{2}=x^{-1} y x y^{-1} x .
$$

Then from the last equality we obtain $x^{-1} y x=y x y^{-1}$, that is, $x y x=y x y$, or also $y^{-1} x y=x y x^{-1}$. Combining this and the first equality of Eq. (7.2), we have $y^{-1} x y=$ $x^{-2} y x^{2}=x y x^{-1}$, which yields $y=x^{-3} y x^{3}$, and so we see that $G\left(4.65^{*}\right)$ is isomorphic to $G_{3}$.

(ii) First, we give a presentation of the commutator subgroup $G_{3}^{\prime}$ of $G_{3}$ by the Reidemeister-Schreier method. Putting $a=y x^{-1}$ and $a_{k}=x^{-k} a x^{k}$, we have

$$
\begin{aligned}
G_{3}^{\prime} & \cong\left\langle a_{k}(k \in \mathbf{Z}) \mid a_{k}=a_{k+1} a_{k-1}, a_{k}=a_{k+3}(k \in \mathbf{Z})\right\rangle \\
& \cong\langle a, b \mid a=b a b, b=a b a\rangle,
\end{aligned}
$$

where $b=a_{1}=x^{-1} y$. This is the quaternion group of order 8 . Thus we have

$$
G_{3} \cong\left\langle x, a, b \mid b=x^{-1} a x, a b=x^{-1} b x, a=b a b, b=a b a\right\rangle ;
$$

see Example 1.1 and Sec. 6 in [14]. 
Let $l$ be the longitude corresponding to the meridian $x$. Then

$$
l=z \cdot x \cdot x \cdot y^{-1} \cdot x^{-2}=x^{-1} y x \cdot x^{2} y^{-1} x^{-2} .
$$

Since the commutator subgroup is also presented by (7.3) or (7.4) in the same words, we have

$$
l=x^{-1} a x x \cdot x^{2}(a x)^{-1} x^{-2}=\left(x^{-1} a x\right)\left(x^{2} a^{-1} x^{-2}\right) a_{1} a_{-2}^{-1}=1,
$$

where $a=y x^{-1}$ and $a_{k}=x^{-k} a x^{k}$.

(iv) From Figs. 12 and 24 we have $\operatorname{Gauß}\left(4.65^{*}\right) \stackrel{\text { ow }}{\sim}-\operatorname{Gauß}(4.96)^{\dagger}$, and so by Proposition 3.4 Tube $\left(4.65^{*}\right) \stackrel{\mathrm{o}}{\approx}$ - Tube(4.96) Since $\mathrm{T} 4_{3}$ is symmetric we obtain the result.

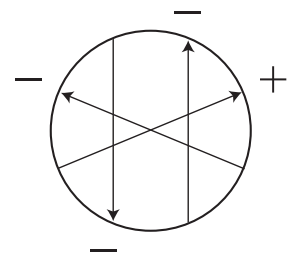

Figure 24. Gauss diagram for the knot 4.96.

Remark 7.2. The ribbon torus knot $\mathrm{T}_{3}$ is equivalent to the ribbon torus knot $F_{1}$ in Example 3.8 of [7], in the form of successive cross-sections.

7.2. Ribbon torus knot $\mathrm{T} 4_{4}$. We define $\mathrm{T} 4_{4}=$ Tube $\left(4.102^{*}\right)$. The knot $4.102^{*}$ is the 2-braid virtual knot $B(-2,1,1)$ as shown in Fig. 25.

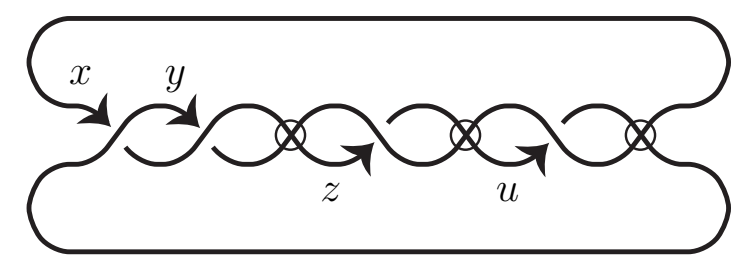

Figure 25. A diagram of the knot $4.102^{*}$, the virtual 2-braid knot $B(-2,1,1)$.

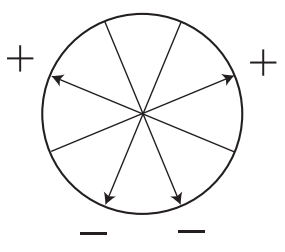

4.102

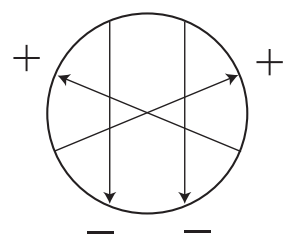

4.104

Figure 26. Gauss diagrams for the knots 4.102, 4.104. 
Lemma 7.3. (i) The ribbon torus knot $\mathrm{T}_{4}$ is symmetric.

(ii) $G\left(\mathrm{~T}_{4}\right) \cong G\left(4.102^{*}\right) \cong G_{3}$.

(iii) The longitude of $\mathrm{T}_{4}$ has order 2 in $G\left(\mathrm{~T} 4_{4}\right)$.

(iv) For $K=4.102,4.104,4.104^{*}, \operatorname{Tube}(K) \stackrel{\mathrm{O}}{\approx} \mathrm{T}_{4}$.

Proof. (i) Green [5] mentions that the virtual knot 4.102 is reversible, and so is $4.102^{*}$, which implies that $\mathrm{T}_{4}$ is symmetric by Proposition 3.4. In fact, from the diagram in Fig. 25 or the Gauss diagram for $4.102^{*}$ (Fig. 26) we see that the knot $4.102^{*}$ is reversible.

(ii) Using the Wirtinger generators $x, y, z, u$ given in Fig. 25 we have

$$
\begin{aligned}
G\left(4.102^{*}\right) & =\left\langle x, y, z, u \mid z=y x y^{-1}, x=z y z^{-1}, u=x^{-1} z x, y=x^{-1} u x\right\rangle \\
& \cong\left\langle x, y, z \mid z=y x y^{-1}, x=z y z^{-1}, y=x^{-2} z x^{2}\right\rangle \\
& \cong\left\langle x, y \mid x=y x y x^{-1} y^{-1}, y=x^{-2} y x y^{-1} x^{2}\right\rangle,
\end{aligned}
$$

where we eliminate the generators $u, z$. Then it is easy to see that $G\left(4.102^{*}\right)$ is isomorphic to $G_{3}$.

(iii) Let $l$ be the longitude corresponding to the meridian $x$. Then

$$
l=y^{-1} \cdot x \cdot x \cdot z^{-1}=\left(x^{-1} a^{-1} x\right)\left(x a x^{-1}\right) a^{-1}=a_{1}^{-1} a_{-1} a^{-1}=b^{2},
$$

where we use the relation in Eq. (7.3). Then the longitude $l$ has order 2 , which is the generator of the center of the quaternion group presented by Eq. (7.4).

(iv) From Fig. 26 we have: $\operatorname{Gauß}(4.102) \stackrel{\text { ow }}{\sim} \operatorname{Gauß}(4.104), \operatorname{Gauß}\left(4.102^{*}\right)=\operatorname{Gauß}\left(4.102^{\dagger}\right)$, and $\operatorname{Gau} ß\left(4.104^{*}\right)=\operatorname{Gau}\left(4.104^{\dagger}\right)$, and thus by Proposition 3.4 we obtain the result.

\section{RibBon torus KNOT With GROUP $G_{7} ; \mathrm{T}_{5}$}

We define $\mathrm{T}_{5}=$ Tube(4.99). Let $J$ be a ribbon 2 -knot given by Fox in $[3$, Example 10]. Note that the orientation of the meridian is taken left-handed. Then we have the following:

Lemma 8.1. (i) $G\left(\mathrm{~T}_{4}\right) \cong G(4.99) \cong G_{7}$.

(ii) $\mathrm{T}_{5}$ is asymmetric.

(iii) For $K=72^{*}, 77^{*}$, $\operatorname{Tube}(K) \stackrel{\mathrm{O}}{\approx} \mathrm{T} 4_{5}$.

(iv) The ribbon torus knot $\mathrm{T}_{5}$ is obtained from Fox's ribbon 2 -knot $J$ by adding a trivial 1-handle.

Proof. (i) Using the generators given in Fig. 27, we have:

$$
G(4.99)=\left\langle x, y, z, u \mid u=z=x^{-1} y x=y^{-1} x y\right\rangle,
$$

which is isomorphic to $G_{7}$.

(ii) Assuming $\mathrm{T} 4_{5}$ is embedded in the 4 -sphere $S^{4}$, let $\tilde{X}$ be the infinite cyclic covering space of the complement $S^{4}-\mathrm{T} 4_{5}$. Then the Alexander invariant $H_{*}(\tilde{X})$ has a $\Lambda$-module 
structure, where $\Lambda$ is the Laurent polynomial ring $\mathbf{Z}\left[t, t^{-1}\right]$; see [25, Chapter 7]. From the group presentation (4.7) of $G_{7}$, we obtain $H_{1}(\tilde{X})=\Lambda /(t-2)$. Since $\left(t^{-1}-2\right) \neq$ $(t-2)$ in $\Lambda, \mathrm{T}_{5}$ is assymetric; cf. [29, Proposition 3.20].

(iii) From Figs. 12 and 28 we have: $\operatorname{Gauß}(4.99) \stackrel{\text { ow }}{\sim} \operatorname{Gauß}\left(4.72^{*}\right) \stackrel{\text { ow }}{\sim} \operatorname{Gauß}\left(4.77^{*}\right)$, and so by Proposition 3.4 we obtain the result.

(iv) The virtual arc diagram given in Fig. 29(a) (Fig. 13 in [26]) presents Fox's 2-knot $J$. Then applying Theorem 5.8 in [26], we obtain the virtual knot diagram given in Fig. 29(b), which presents a ribbon torus knot obtained from $J$ by adding a trivial 1-handle. Since its Gauss diagram is the same as that of $4.77^{*}$, the proof is complete.

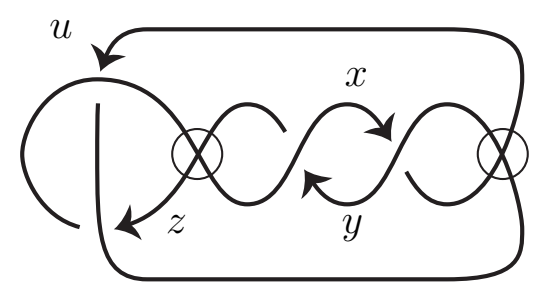

Figure 27. A diagram of the knot 4.99.

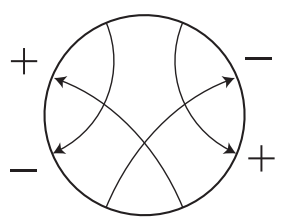

$4.72^{*}$

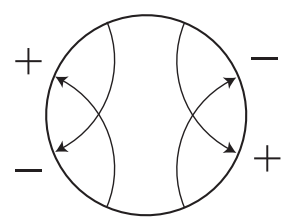

$4.77^{*}$

Figure 28. Gauss diagrams for the knots $4.72^{*}, 4.77^{*}$.

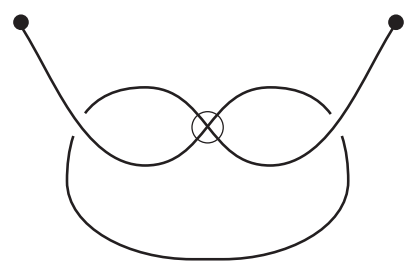

(a)

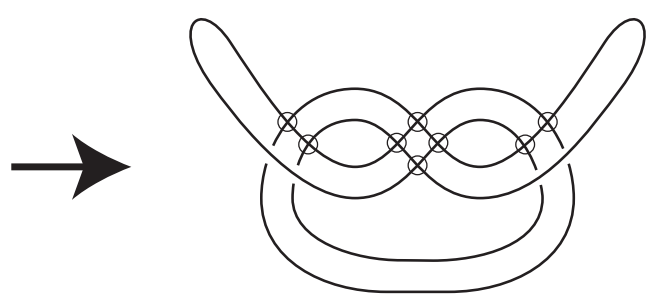

(b)

Figure 29. The virtual arc diagram presenting Fox's 2-knot $J$ yields the knot $4.77^{*}$. 
Remark 8.2. Putting $y=a x$ in the presentation (4.7), we have

$$
G_{7} \cong\left\langle x, a \mid x a x^{-1}=a^{2}\right\rangle .
$$

Putting $a_{k}=x^{-k} a x^{k}$, by the Reidemeister-Schreier method we have the presentation of the commutator subgroup $G_{7}^{\prime}$ of $G_{7}$.

$$
G_{7}^{\prime}=\left\langle a_{k}(k \in \mathbf{Z}) \mid a_{k}=a_{k+1}^{2}(k \in \mathbf{Z})\right\rangle .
$$

which is the additive group of the dyadic rationals $\mathbf{Z}[1 / 2]$.

\section{RibBon torus knot with group $G_{8} ; \mathrm{T}_{6}$}

We define $\mathrm{T} 4_{6}=\operatorname{Tube}\left(4.62^{*}\right)$.

Lemma 9.1. (i) $G\left(\mathrm{~T}_{6}\right) \cong G\left(4.62^{*}\right) \cong G_{8}$.

(ii) $\mathrm{T}_{6}$ is asymmetric.

(iii) - Tube $(4.95) \stackrel{ }{\approx}$ Tube $\left(4.95^{*}\right) \stackrel{\circ}{\approx} \mathrm{T} 4_{6}$.

Proof. (i) The group of the knot $4.62^{*}$ as shown in Fig. 30 has the presentation:

$$
\begin{aligned}
G\left(4.62^{*}\right) & =\langle x, y, z, u \mid z x=x u=u y, u z=x y=y u\rangle \\
& \cong\left\langle x, y \mid x y^{-1} x=y^{-1} x y, y^{-1} x^{-1} y x y x=x y^{-1} x y\right\rangle .
\end{aligned}
$$

Putting $a=y x^{-1}$ and $a_{k}=x^{-k} a x^{k}$, by the Reidemeister-Schreier method we obtain the presentation of the commutator subgroup:

$$
\begin{aligned}
& \left\langle a_{k}(k \in \mathbf{Z}) \mid a_{k}^{2}=a_{k+1}, a_{k} a_{k-2}=a_{k-2} a_{k-3}(k \in \mathbf{Z})\right\rangle \\
\cong & \left\langle a_{0} \mid a_{0}^{7}=1\right\rangle \cong \mathbf{Z} / 7 \mathbf{Z},
\end{aligned}
$$

Since $x^{-1} a x=a_{1}=a^{2}$, we obtain the presentation (4.8) of $G_{8}$.

(ii) As in the proof of Lemma 8.1(iii), assuming $\mathrm{T}_{6}$ is embedded in the 4 -sphere $S^{4}$, let $\tilde{X}$ be the infinite cyclic covering space of the complement $S^{4}-\mathrm{T} 4_{6}$. Then from the group presentation (4.8) of $G_{8}$, we obtain $H_{1}(\tilde{X})=\Lambda /(t-2,7)$. Regarding this as a $\Lambda_{7}$-module, we have $H_{1}(\tilde{X})=\Lambda_{7} /(t-2)$, where $\Lambda_{7}=\Lambda /(7)$. If $\mathrm{T}_{6}$ were symmetric, then $\Lambda_{7} /(t-2)$ and $\Lambda_{7} /\left(t^{-1}-2\right)$ should be isomorphic, which is a contradiction since $(t-2) \neq\left(t^{-1}-2\right)$ in the principal ideal domain $\Lambda_{7}$.

(iii) From Figs. 12 and 31 we have $\operatorname{Gau} \beta(4.95)^{\dagger} \stackrel{\text { ow }}{\sim}-\operatorname{Gau} \beta\left(4.62^{*}\right)$ and $\operatorname{Gauß}\left(4.95^{*}\right) \stackrel{\text { ow }}{\sim} \operatorname{Gauß}\left(4.62^{*}\right)$, which imply Tube $(4.95) \stackrel{\mathrm{O}}{\approx}-\mathrm{T} 4_{6}$ and Tube $\left(4.95^{*}\right) \stackrel{\mathrm{O}}{\approx} \mathrm{T} 4_{6}$ by Proposition 3.4.

Remark 9.2. The group $G_{8}$ is not a 2 -knot group, but a 3-knot group; see [18, Theorem 14.1.7], where $G_{8}$ is realized as a group of a 3 -knot which is obtained by 3-twist spinning from the Fox's ribbon 2-knot $J$ [3, Example 10] with group $G_{7}$; see Sec. 8. 


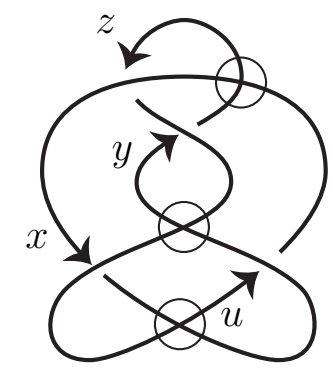

FiguRe 30 . A diagram of the knot $4.62^{*}$.

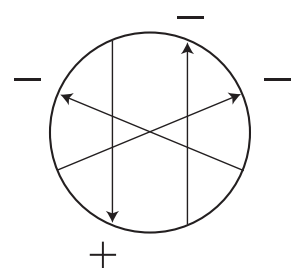

FiguRE 31. Gauss diagram for the knot 4.95.

10. Ribbon torus knot With group $G_{9} ; 4 \mathrm{~T}_{7}$

We define $4 \mathrm{~T}_{7}=$ Tube(4.105).

Lemma 10.1. (i) $G\left(\mathrm{~T} 4_{7}\right) \cong G(4.105) \cong G_{9}$.

(ii) $\mathrm{T}_{7}$ is asymmetric.

(iii) - Tube $\left(4.105^{*}\right) \stackrel{\mathrm{O}}{\approx} \mathrm{T}_{7}$.

Proof. (i) The group of the knot 4.105 as shown in Fig. 32 has the presentation:

$$
\begin{aligned}
G(4.105) & =\left\langle x, y, z, u \mid u=y x y^{-1}, z=u^{-1} y u, y=z^{-1} x z, z=x u x^{-1}\right\rangle \\
& \cong\left\langle x, y, z \mid z=y x^{-1} y x y^{-1}, y=z^{-1} x z, z=x y x y^{-1} x^{-1}\right\rangle .
\end{aligned}
$$

Removing $z$, we obtain the presentation (4.9) of $G_{9}$.

(ii) The proof is similar to that of Lemma 8.1(iii). We use $H_{1}(\tilde{X})=\Lambda /\left(t^{2}-2 t+2\right)$, where $\tilde{X}$ is the infinite cyclic covering space of the complement $\mathrm{T}_{7}$ in $S^{4}$.

(iii) From Fig. 12 Gauß $\left(4.105^{*}\right)=-\operatorname{Gauß}(4.105)^{\dagger}$, which implies the result by Proposition 3.4. This completes the proof.

The group $G_{9}$ has the following feature.

Proposition 10.2. The commutator subgroup of $G_{9}$ is abelian.

Proof. First we give a presentation of the commutator subgroup $G_{9}^{\prime}$ from the presentation (4.9) of $G_{9}$ by the Reidemeister-Schreier method. Putting $a=y x^{-1}$ and 


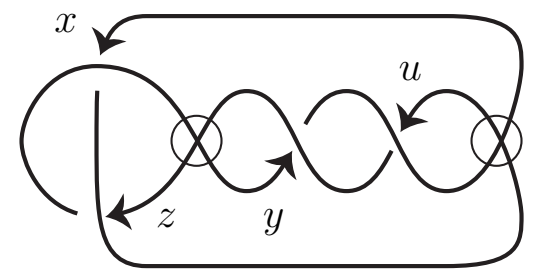

FiguRE 32. A diagram of the knot 4.105.

$a_{k}=x^{-k} a x^{k}$, we obtain:

$$
\begin{aligned}
G_{9}^{\prime} & \cong\left\langle a_{k}(k \in \mathbf{Z}) \mid a_{k}^{2} a_{k+1}^{-1}=a_{k+1} a_{k+2}^{-1}=a_{k} a_{k+1}^{-1} a_{k}(k \in \mathbf{Z})\right\rangle \\
& \cong\left\langle a_{k}(k \in \mathbf{Z}) \mid a_{k+2}=a_{k}^{-2} a_{k+1}^{2}, a_{k} a_{k+1}=a_{k+1} a_{k}(k \in \mathbf{Z})\right\rangle .
\end{aligned}
$$

We show that any two generators $a_{i}$ and $a_{j}$ commute, where $i<j$. By induction we may show $a_{j}=a_{i}^{m_{j}} a_{i+1}^{n_{j}}$ for some integers $m_{j}, n_{j}$. Then since $a_{i}$ and $a_{i+1}$ commute, $a_{i}$ and $a_{j}$ commute. This completes the proof.

Remark 10.3. Hillman [6, Theorem 14 and Corollary in p. 144] has determined the abelian 2-knot commutator subgroups; cf. [21, 33]: If $G$ is a 2-knot group whose commutator subgroup is abelian, then either $G^{\prime} \cong \mathbf{Z}^{3}$ or $\mathbf{Z} / n \mathbf{Z}$ for some odd integer $n$, or $G \cong G_{7}$, where $G_{7}$ is presented by (4.7); see Remark 8.2. Therefore, $G_{9}$ is not a 2-knot group. In fact, $\mathrm{T} 4_{5}$ and $\mathrm{T} 4_{7}$ have different Alexander invariants. However, we do not know whether $G_{9}^{\prime}$ is isomorphic to $\mathbf{Z}[1 / 2]\left(\cong G_{7}^{\prime}\right)$ or not.

\section{Ribbon torus knots With group $G_{5} ; \mathrm{T} 4_{8}, \mathrm{~T} 4_{9}$}

There are at most two ribbon torus knots with group $G_{5}$ represented by virtual knots with up to 4 crossings; $\mathrm{T} 4_{8}, \mathrm{~T} 4_{9}$. However, we do not know whether they are equivalent or not.

11.1. Ribbon torus knot $\mathrm{T} 4_{8}$. We define $\mathrm{T} 4_{8}=$ Tube(4.106).

Lemma 11.1. (i) $G\left(\mathrm{~T}_{8}\right) \cong G(4.106) \cong G_{5}$.

(ii) $\mathrm{T}_{8}$ is symmetric.

(iii) Tube $\left(4.106^{*}\right) \stackrel{\mathrm{o}}{\approx} \mathrm{T}_{8}$.

Proof. (i) The group of the knot 4.106 as shown in Fig. 33 has the presentation:

$$
\begin{aligned}
G(4.106) & =\langle x, y, z, u \mid y u=u x, y z=u y, u z=z x, x y=z x\rangle \\
& \cong\left\langle x, y \mid y x y x y^{-1} x^{-1}=x y x y^{-1}, y x y x^{-1}=x y x y^{-1} x^{-1} y\right\rangle .
\end{aligned}
$$


Putting $a=y x^{-1}$ and $a_{k}=x^{-k} a x^{k}$, by the Reidemeister-Schreier method we obtain the presentation of the commutator subgroup:

$$
\begin{aligned}
& \left\langle a_{k}(k \in \mathbf{Z}) \mid a_{k+3} a_{k+1} a_{k}^{-1}=a_{k+2} a_{k+1}^{-1}, a_{k+2} a_{k}=a_{k+1} a_{k}^{-1} a_{k+1}(k \in \mathbf{Z})\right\rangle \\
\cong & \left\langle a_{k}(k \in \mathbf{Z}) \mid a_{0}^{5}=1, a_{k}=a_{k+1}^{-1}(k \in \mathbf{Z})\right\rangle \cong \mathbf{Z} / 5 \mathbf{Z},
\end{aligned}
$$

from which we obtain the presentation (4.5) of $G_{5}$.

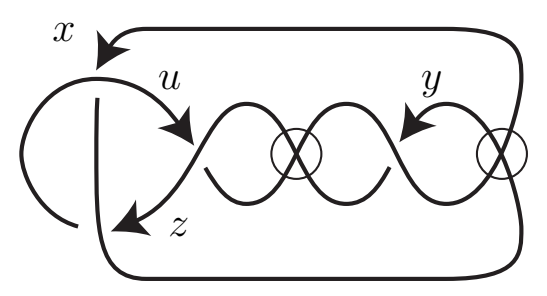

Figure 33. A diagram of the knot 4.106.

(ii) Green [5] mentions that 4.106 is reversible, which implies that $\mathrm{T} 4_{8}$ is symmetric. In fact, we have another diagram of 4.106 as shown in Fig. 34 indicating the reversibility; rotating the whole diagram 180 degrees, we obtain the reversion.

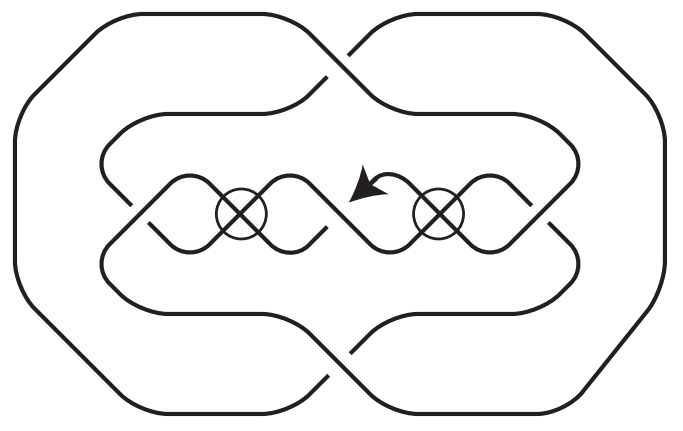

FiguRE 34. Another diagram of the knot 4.106 indicating the reversibility.

(iii) From Fig. $12 \operatorname{Gau}\left(4.106^{*}\right)=-\operatorname{Gau}(4.106)^{\dagger}$. Since $\mathrm{T} 4_{8}$ is symmetric, by Propsition 3.4 we obtain the result. This completes the proof.

11.2. Ribbon torus knot $\mathrm{T} 4_{9}$. We define $\mathrm{T} 4_{9}=$ Tube(4.107).

Lemma 11.2. (i) $G\left(\mathrm{~T}_{9}\right) \cong G(4.107) \cong G_{5}$.

(ii) $\operatorname{Tube}\left(4.107^{*}\right) \stackrel{\mathrm{o}}{\approx}-\mathrm{T} 4_{9}$.

Proof. (i) The group of the knot 4.107 as shown in Fig. 35 has the presentation:

$$
\begin{aligned}
G(4.107) & =\langle x, y, z, u \mid y x=u y=z u, z y=x z=u x\rangle \\
& \cong\left\langle x, y \mid x^{-1} y x y^{-1} x=y x y x^{-1} y^{-1}=y x y^{-1} x y^{-1}\right\rangle .
\end{aligned}
$$


From the last relation, we obtain $x^{2}=y^{2}$, which is in the center of this group, and so we have:

$$
G(4.107) \cong\left\langle x, y \mid x y x y x=y x y x y, x^{2}=y^{2}\right\rangle .
$$

Putting $a=y x^{-1}$ and $a_{k}=x^{-k} a x^{k}$, by the Reidemeister-Schreier method we obtain the presentation of the commutator subgroup:

$$
\left\langle a_{k}(k \in \mathbf{Z}) \mid a_{k}^{5}=1, a_{k+1}=a_{k}(k \in \mathbf{Z})\right\rangle \cong \mathbf{Z} / 5 \mathbf{Z},
$$

from which we obtain the presentation (4.5) of $G_{5}$.

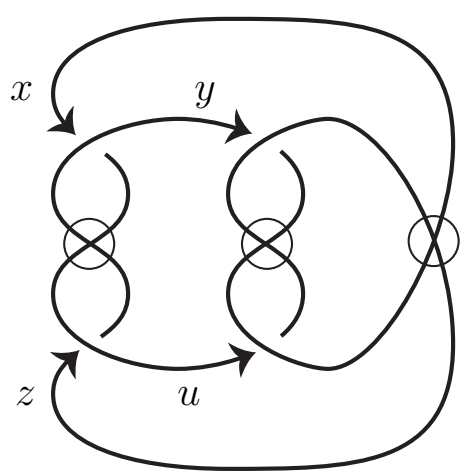

FiguRe 35. A diagram of the knot 4.107.

(ii) From Fig. 12 Gauß $\left.4.107^{*}\right)=-$ Gauß(4.107), which implies the result by Proposition 3.4. This completes the proof.

Remark 11.3. We do not know whether $\mathrm{T} 4_{9}$ is symmetric or not. According to Green [5] the knot 4.107 is not reversible.

\section{Ribbon torus knots With group $G_{6} ; \mathrm{T} 4_{10}, \mathrm{~T}_{11}$}

There are at most two ribbon torus knots with group $G_{6}$ represented by virtual knots with up to 4 crossings; $\mathrm{T}_{10}, \mathrm{~T}_{11}$. However, we do not know whether they are equivalent or not.

12.1. Ribbon torus knot $\mathrm{T} 4_{10}$. We define $\mathrm{T} 4_{10}=$ Tube $\left(4.66^{*}\right)$.

Lemma 12.1. (i) $G\left(\mathrm{~T}_{10}\right) \cong G\left(4.66^{*}\right) \cong G_{6}$.

(ii) $\mathrm{T}_{10}$ is asymmetric.

(iii) - Tube $(4.97) \stackrel{\stackrel{0}{\approx}}{\mathrm{T}} 4_{10}$.

Proof. (i) The group of the knot $4.66^{*}$ as shown in Fig. 36 has the presentation:

$$
\begin{aligned}
& G\left(4.66^{*}\right)=\langle x, y, z, u \mid x z=z y, u y=z u, u z=z x, x z=u x\rangle \\
& \cong\left\langle x, y \mid z x z^{-1}=x z x^{-1}, z^{-1} x z=x z^{-1} x^{-1} z x z x^{-1}\right\rangle . \\
& 25
\end{aligned}
$$


Putting $a=z x^{-1}$ and $a_{k}=x^{-k} a x^{k}$, by the Reidemeister-Schreier method we obtain the presentation of the commutator subgroup:

$$
\begin{aligned}
& \left\langle a_{k}(k \in \mathbf{Z}) \mid a_{k+1}=a_{k}^{2}, a_{k+1}^{-1} a_{k}=a_{k}^{-1} a_{k+1} a_{k-1}(k \in \mathbf{Z})\right\rangle \\
& \left\langle a_{k}(k \in \mathbf{Z}) \mid a_{k+1}=a_{k}^{2}, a_{k}^{5}=1(k \in \mathbf{Z})\right\rangle \cong \mathbf{Z} / 5 \mathbf{Z},
\end{aligned}
$$

from which we obtain the presentation (4.6) of $G_{6}$.

(ii) The proof is similar to that of Lemma 9.1(ii). We use $H_{1}(\tilde{X})=\Lambda /(5, t-2)$, where $\tilde{X}$ is the infinite cyclic covering space of the complement T4 $4_{10}$ in $S^{4}$.

(iii) From Figs. 12 and $37 \operatorname{Gauß}\left(4.66^{*}\right) \stackrel{\text { ow }}{\sim}-\operatorname{Gauß}(4.97)^{\dagger}$, which implies the result by Proposition 3.4. This completes the proof.

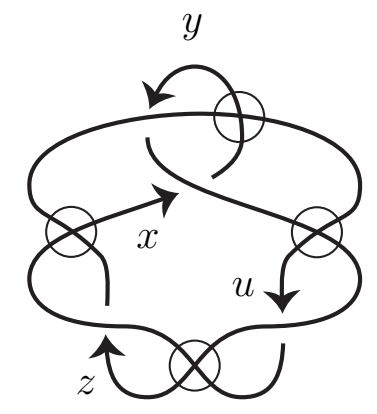

Figure 36. A diagram of the knot $4.66^{*}$.

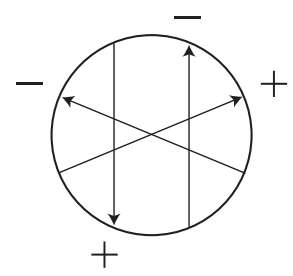

Figure 37. Gauss diagram for the knot 4.97.

12.2. Ribbon torus knot $\mathrm{T} 4_{11}$. We define $\mathrm{T} 4_{11}=$ Tube(4.101).

Lemma 12.2. (i) $G\left(\mathrm{~T}_{11}\right) \cong G_{6}$.

(ii) $\mathrm{T}_{11}$ is asymmetric.

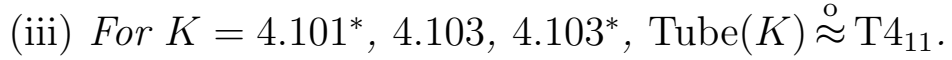

Proof. (i) The virtual 2-braid knot $B(-2,-1,1)$ with its orientation reversed is a diagram of the knot 4.101 as shown in Fig. 38. In [14, Example 3.2] it is shown that the group of the knot $B(-2,-1,1)$ is isomorphic to $G_{6}$, and so $G\left(\mathrm{~T} 4_{11}\right) \cong G\left(-\mathrm{T} 4_{11}\right) \cong G_{6}$.

(ii) The proof is the same as that of Lemma 12.1(ii).

(iii) Green [5] mentions that $4.101^{*} \sim 4.101^{\dagger}$. In fact, Fig. 12 shows $\operatorname{Gauß}(4.101)^{*}=$

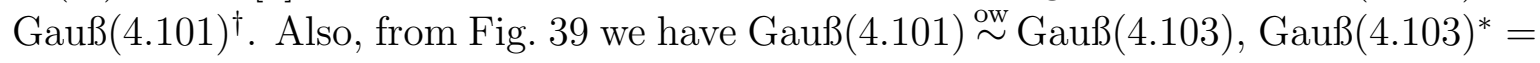


Gauß(4.103) ${ }^{\dagger}$. Then by Proposition 3.4 we obtain the result. This completes the proof.

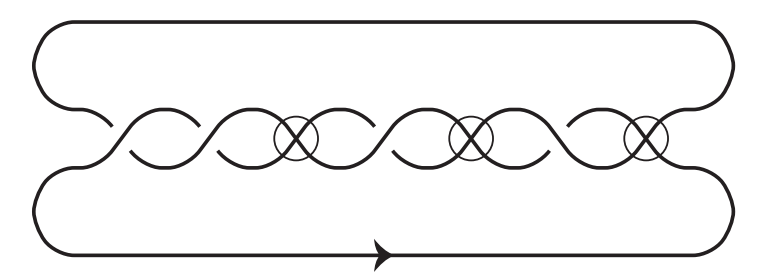

Figure 38. A diagram of the knot 4.101.

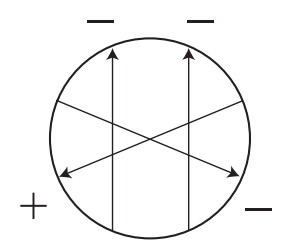

Figure 39. Gauss diagram for the knot 4.103.

Remark 12.3. The group $G_{6}$ is not a 2 -knot group, but a 3 -knot group; cf. [18, Exercise 14.1.8].

\section{Remarks And Questions}

First we give some remarks.

Remark 13.1. Winter [30, Theorem 3.7] has remarked that the Tube operation is not injective: Let $K$ be the right-hand trefoil knot. Then $K$ and $K^{*}$, the left-hand trefoil knot, are not equivalent as oriented welded virtual knots. Since $K^{*}=-K^{\dagger}$, by Eq. (3.5) in Proposition 3.3 we have $\operatorname{Tube}(K) \stackrel{\mathrm{o}}{\approx} \operatorname{Tube}\left(K^{*}\right)$.

Remark 13.2. Silver and Williams [28, Theorem 2.2] have given a characterization of virtual knot groups: A virtual knot group $G$ is isomorphic to the group of some ribbon torus knot. This gives Satoh's Tube operation algebraically.

Remark 13.3. Maeda [23, Lemma 3.3.2] has shown that if a finitely presented group $G$ satisfies $G / G^{\prime} \cong \mathbf{Z}$ and $G^{\prime} \cong \mathbf{Z} / m \mathbf{Z}, m$ is a positive integer, then $G$ is isomorphic to the group

$$
\left\langle x, a \mid x^{-1} a x=a^{n}, a^{m}=1\right\rangle
$$

for some integer $n$ with $(m, n)=(n-1, m)=1$. The groups $G_{2}$ (Eq. (6.2)), $G_{5}, G_{6}$, $G_{8}$ are such groups. Maeda proved that putting $y=x a^{-1}$, the group $G$ becomes:

$$
\left\langle x, y \mid y=\left(y^{-1} x\right)^{-q} x\left(y^{-1} x\right)^{q}, x=\left(y^{-1} x\right)^{-m} x\left(y^{-1} x\right)^{m}\right\rangle,
$$


where $q(n-1)+r m=1(q, r \in \mathbf{Z})$. From this presentation we can easily see that $G$ is the group of some ribbon torus knot, or equivalently, of some virtual knot. This may be also proved by using Corollary 2.3 in [28]. Further, if $G$ is a 2-knot group, then $n=-1[6,21]$.

From Theorem 4.1 and Remark 11.3 the following is open:

Question 13.4. (i) Decide whether each pair of the ribbon torus knots $\left\{\mathrm{T} 4_{8}, \mathrm{~T} 4_{9}\right\}$, $\left\{\mathrm{T} 4_{10}, \mathrm{~T} 4_{11}\right\}$ are equivalent or not.

(ii) Is the ribbon torus knot $\mathrm{T} 4_{9}$ symmetric? Note that $\mathrm{T} 4_{8}$ is symmetric.

We should consider the remaining virtual knots; knots whose groups are infinite cyclic. Many such knots are easily seen to be oriented welded-equivalent to the trivial knot by using deformations of Gauss diagrams just like the knot 3.1 in Example 2.4, where we use only the moves $\Omega_{1}$ and $\Phi_{t}$, and some are deformed into the trivial knot by the generalized Reidemeister moves and the move $\Phi_{t}$ on virtual knot diagrams. There also remaining a few cases we cannot deform into the trivial knot as Question 13.4(i). They presumably present trivial ribbon torus knots. So in order to show this, we might need some other moves than $\Phi_{t}$ for a welded virtual knot. So we may ask the following question, which relates to Winter's example in Remark 13.1.

Question 13.5. Suppose that two virtual knots $K$ and $K^{\prime}$ represent the same ribbon torus knot; $\operatorname{Tube}(K) \stackrel{\mathrm{o}}{\approx} \operatorname{Tube}\left(K^{\prime}\right)$. Find a relationship between $K$ and $K^{\prime}$.

\section{REFERENCES}

[1] A. M. Brunner, E. J. Mayland, and J. Simon, Knot groups in $S^{4}$ with nontrivial homology, Pacific J. Math. 103 (1982) 315-324.

[2] R. Fenn, R. Rimanyi and C. Rourke, The braid-permutation group, Topology 36 (1997) 123-135.

[3] R. H. Fox, A quick trip through knot theory, In: Topology of 3-manifolds and related topics (Georgia, 1961), 120-167 (1962) Prentice-Hall.

[4] M. Goussarov, M. Polyak, and O. Viro, Finite-type invariants of classical and virtual knots, Topology 39 (2000) 1045-1068.

[5] J. Green, A table of virtual knots, http://www.math.toronto.edu/drorbn/Students/GreenJ/

[6] J. A. Hillman, Abelian normal subgroups of two-knot groups, Comment. Math. Helvetici 61 (1986) $122-148$.

[7] F. Hosokawa, T. Maeda and S. Suzuki, Numerical invariants of surfaces in 4-space, Math. Sem. Notes Kobe Univ. 7 (1979), 409-420.

[8] D. Hrencecin and L. H. Kauffman, Biquandles for virtual knots, J. Knot Theory Ramifications 16 (200) 1361-1382.

[9] A. Ichimori, Ribbon torus knot presented by virtual knots, Master's Thesis (in Japanese), Osaka City University (2011).

[10] T. Kanenobu, Recursive calculation for an invariant of a ribbon knot, J. Knot Theory Ramifications 7 (1998) 1093-1105.

[11] T. Kanenobu, An unknotting operation on ribbon 2-knots, J. Knot Theory Ramifications 9 (2000) 1011-1028.

[12] T. Kanenobu, Forbidden moves unknot a virtual knot, J. Knot Theory Ramifications 10 (2001) 89-96. 
[13] T. Kanenobu and A. Shima, Two filtrations of ribbon 2-knots, Topology Appl. 121 (2002) 143168.

[14] T. Kanenobu and K. Tsuji, Groups of two-braid virtual knots, J. Knot Theory Ramifications 16 (2007) 671-697.

[15] L. H. Kauffman, Virtual knot theory, Europ. J. Combin. 20 (1999) 663-690.

[16] L. H. Kauffman, A survey of virtual knot theory, in "Knots in Hellas '98, Proceedings of the Conference on Knot Theory and its Ramifications (Delphi)", (C. McA. Gordon, V. F. R. Jones, L. Kauffman and J. H. Przytycki, eds.) Series on Knots and Everything, Vol. 24, World Scientific Publ. Co., (2000), 143-202.

[17] L. H. Kauffman and J. Faria Martins, Invariants of welded virtual knots via crossed module invariants of knotted surfaces, Compositio Math. 144 (2008) 1046-1080.

[18] A. Kawauchi, A Survey of Knot Theory, Birkhäuser, Basel (1996).

[19] S.-G. Kim, Virtual knot groups and their peripheral structure, J. Knot Theory Ramifications 9 (2000) 797-812.

[20] T. Kishino and S. Satoh, A note on non-classical virtual knots, J. Knot Theory Ramifications 13 (2004) 845-856.

[21] J. Levine, Some results on higher dimensional knot groups, Lecture Notes in Math., vol. 685, Springer-Verlag, Berlin and New York, 1978, 243-269.

[22] C. Livingston, Stably irreducible surfaces in $S^{4}$, Pacific J. Math. 116 (1985) 77-84.

[23] T. Maeda, Knot groups as commutator extension, Master Thesis (in Japanese), Kwansei Gakuin Univ. (1977).

[24] S. Nelson, Unknotting virtual knots with Gauss diagram forbidden moves, J. Knot Theory Ramifications 10 (2001) 931-935.

[25] D. Rolfsen, Knots and Links, (1976) Publish or Perish.

[26] S. Satoh, Virtual knot presentation of ribbon torus-knots, J. Knot Theory Ramifications 9 (2000) $531-542$. .

[27] J. Sawollek, An orientation-sebsitive Vassiliev invariant for virtual knots, J. Knot Theory Ramifications 12 (2003) 767-779.

[28] D. S. Silver and S. G. Williams, Virtual knot groups, in "Knots in Hellas '98, Proceedings of the Conference on Knot Theory and its Ramifications (Delphi)", (C. McA. Gordon, V. F. R. Jones, L. Kauffman and J. H. Przytycki, eds.) Series on Knots and Everything, Vol. 24, World Scientific Publ. Co., (2000), 440-451.

[29] S. Suzuki, Knotting problems of 2-spheres in the 4-sphere, Math. Sem. Notes Kobe Univ. 4 (1976) 241-371.

[30] B. Winter, The classification of spun torus knots, J. Knot Theory Ramifications 18 (2009) 12871298.

[31] B. Winter, On the classification problem for ribbon torus knots, J. Knot Theory Ramifications (to appear); math.GT/0711.1638.

[32] T. Yajima, On simply knotted spheres in $R^{4}$, Osaka J. Math. 1 (1964) 133-152.

[33] K. Yoshikawa, On 2-knot groups with abelian commutator subgroups, Proc. Amer. Math. Soc. 92 (1984) 305-310.

[34] E. C. Zeeman, Twisting spun knots, Trans. Amer. Math. Soc. 115 (1965) 471-495. 
Department of Mathematics, Osaka City University, Sugimoto, Sumiyoshi-ku, Osaka 558-8585, JAPAN

E-mail address: m09sa002@ex.media.osaka-cu.ac.jp

Department of Mathematics, Osaka City University, Sugimoto, Sumiyoshi-ku, Osaka 558-8585, JAPAN

E-mail address: kanenobu@sci.osaka-cu.ac.jp 